Article

\title{
Exploring the In Vivo Existence Forms (23 Original Constituents and 147 Metabolites) of Astragali Radix Total Flavonoids and Their Distributions in Rats Using HPLC-DAD-ESI-IT-TOF-MS ${ }^{\mathbf{n}}$
}

\author{
Li-Jia Liu, Hong-Fu Li, Feng Xu * , Hong-Yan Wang, Yi-Fan Zhang, Guang-Xue Liu, \\ Ming-Ying Shang, Xuan Wang and Shao-Qing Cai * \\ State Key Laboratory of Natural and Biomimetic Drugs, School of Pharmaceutical Sciences, Peking University, \\ No. 38 Xueyuan Road, Beijing 100191, China; lijialiu@bjmu.edu.cn (L.-J.L.); 1716383012@bjmu.edu.cn (H.-F.L.); \\ wanghy@pku.org.cn (H.-Y.W.); zhangyf0911@pku.org.cn (Y.-F.Z.); guangxl@bjmu.edu.cn (G.-X.L.); \\ myshang@bjmu.edu.cn (M.-Y. S.); xuanwang6818@bjmu.edu.cn (X.W.) \\ * Correspondence: xufeng76@bjmu.edu.cn (F.X.); sqcai@bjmu.edu.cn (S.-Q.C.); \\ Tel.: +86-10-8280-2534 (F.X.); +86-10-8280-1693 (S.-Q.C.)
}

Received: 1 November 2020; Accepted: 23 November 2020; Published: 26 November 2020

\begin{abstract}
Astragali Radix total flavonoids (ARTF) is one of the main bioactive components of Astragali Radix (AR), and has many pharmacological effects. However, its metabolism and effective forms remains unclear. The HPLC-DAD-ESI-IT-TOF-MS ${ }^{n}$ technique was used to screen and tentatively identify the in vivo original constituents and metabolites of ARTF and to clarify their distribution in rats after oral administration. In addition, modern chromatographic methods were used to isolate the main metabolites from rat urine and NMR spectroscopy was used to elucidate their structures. As a result, 170 compounds (23 original constituents and 147 metabolites) were tentatively identified as forms existing in vivo, 13 of which have the same pharmacological effect with ARTF. Among 170 compounds, three were newly detected original constituents in vivo and 89 were new metabolites of ARTF, from which 12 metabolites were regarded as new compounds. Nineteen original constituents and 65 metabolites were detected in 10 organs. Four metabolites were isolated and identified from rat urine, including a new compound (calycoisn-3'-O-glucuronide methyl ester), a firstly-isolated metabolite (astraisoflavan-7-O-glucoside-2'-O-glucuronide), and two known metabolites (daidzein-7-O-sulfate and calycosin-3'-O-glucuronide). The original constituents and metabolites existing in vivo may be material basis for ARTF efficacy, and these findings are helpful for further clarifying the effective forms of ARTF.
\end{abstract}

Keywords: Astragali Radix; flavonoids; metabolism; LC-MS; effective forms

\section{Introduction}

Astragali Radix total flavonoids (ARTF) is one of the main bioactive components of Astragali Radix (AR, Huangqi in Chinese) [1]. Many studies have proven its cardiovascular protective effect, owing that it exhibited a protective effect on an ischemia-reperfusion model by effectively inhibiting the free radical spectrum [2,3], and exhibited vasorelaxant and endothelial-protective effect via the Akt/eNOS signaling pathway [4]. ARTF has an obvious protective effect on the inflammatory response in brain tissue of a natural aging rat by reducing the expression level of the downstream inflammatory factors [5]. ARTF also has immunostimulatory and anti-inflammatory effects via regulating MAPK (Mitogen-Activated Protein Kinase) and NF-KB signaling pathways [6,7]. ARTF has a protective effect 
against hepatic damage induced by paracetamol [8] or reperfusion [9] as well. In a word, ARTF has a wide range of pharmacological actions.

Up to now, besides studies on the pharmacological actions of AR, many investigations have been conducted in the field of phytochemistry. Over 70 flavonoid compounds have been isolated and identified from AR by modern chromatographic and spectroscopic methods [10-12], and 421 flavonoids have been detected and characterized from AR (the roots of Astragalus membranaceus (Fisch.) Bge. var. mongholicus (Bge.) Hsiao) by HPLC-MS (High Performance Liquid Chromatography-Mass Spectrometry) technology [13]. All these findings indicate that many kinds of constituents exist in ARTF. The metabolism of some high content flavonoid compounds in ARTF have been reported by our research group. Forty-one and 21 metabolites have been identified from the urine of rats after administration of calycosin-7-O-glucoside [14] and ononin [15], respectively. Twenty-six and 14 metabolites have been identified when calycosin [14] and formononetin [15] were incubated with rat liver S9. Relatively, the metabolic studies of isoflavan and pterocarpan are pretty few, and only our research group has done several before. Twenty-one and 20 metabolites of astrapterocarpan-3-O-glucoside, astraisoflavan-7-O-glucoside have been identified in rats, respectively [15]. Forty and 19 metabolites of astrapterocarpan [16] and astraisoflavan [15] have been detected when incubated with rat liver S9, respectively. And many other research groups also have identified some metabolites of these four main isoflavones (calycosin [17], calycosin-7-O-glucoside [18], formononetin [19,20], ononin [21]) in different test systems, such as human gut microbiota [18], zebrafish larvae [17], sheep [19], human liver microsome [20,21], and so on.

However, the forms existing in vivo (i.e., absorbed constituents and metabolites) of ARTF remains unclear. Our research group had done a study on ARTF metabolism before, and identified 127, 43, and 22 compounds in the urine, plasma, and feces, respectively [22]. But in that study, no glucuronides of ARTF were found, which should be main metabolites of flavonoid compounds; the distribution of ARTF was not investigated either. And the dose schedule of ARTF was once per day for seven days. As is well known, it usually needs a long dose period for traditional Chinese medicines to treat diseases in clinical practice. As for AR, it can be used for a long time [23]. Therefore, to better simulate the long dose period of AR and understand the existence forms in vivo of ARTF under long-term administration situation, this study was performed. The rats were orally administrated with ARTF twice a day for 31 days, and the samples of urine, plasma, feces, and organs were collected to identify original constituents and metabolites that existed in vivo, and to study the distribution of the existence forms in vivo. After that, the ARTF-containing urine was used to isolate some major metabolites.

\section{Results and Discussion}

To know which compounds exist in vivo, which is one of the prerequisites for the compounds to be effective forms, the compounds in the bio-samples including urine, plasma, feces, and organs of the rats after oral administration of ARTF were analyzed. In total, one hundred and seventy compounds (23 original constituents and 147 metabolites) were identified, among which 12 were regarded as new compounds (they are all metabolites) by retrieving information from the SciFinder database, three were newly detected original constituents, and 89 were new metabolites of ARTF. In 147 metabolites, nine were phase I and 138 were phase II metabolites. Among 138 phase II metabolites, ninety-two sulfates, twenty-six glucuronides, thirteen both sulfuric acid, and glucuronic acid conjugates and seven methyl conjugates were included, which indicated that sulfates were the most important existence forms of ARTF. To better understand the distribution of compounds, ten different organs of the rat were analyzed as well. Nineteen original constituents including six ones detected only in the organs (F18-F23) and 65 metabolites containing three ones detected only in the organs (M145-M147) were identified. Three, 5, 4, 3, 5, 17, 7, 7, 6 original constituents together with 3, 18, 5, 6, 22, 30, 46, 21, 6 metabolites were identified in the heart, liver, spleen, lungs, kidneys, stomach, small intestine, colon intestine, and thymus, respectively. However, no compounds were found in the brain. Four metabolites were isolated from the ARTF-containing urine and identified by NMR. To better understand potential 
effective forms among those compounds in vivo, we retrieved over 40 compounds which have specific structures from SciFinder, and 13 compounds including six original constituents, and seven metabolites were found to possess the same pharmacological effects as ARTF. Most of the phase II metabolites were not found to possess bioactivity, perhaps owing that this kind of material was difficult to gain and to study.

\subsection{Identification of Original Constituents and Metabolites of ARTF in Rats}

\subsubsection{Identification of Original Constituents}

The peaks which appeared at the same position in LC-MS chromatograms of both the administrated bio-samples and the ARTF but did not exist in the blank bio-samples were regarded as original constituents. By comparison of their extracted ion chromatograms (EICs) and base peak chromatograms (BPCs), twenty-three original constituents were identified (Table 1, F1-F23, and F18-F23 only detected in the organs). They were composed of 15 flavones or isoflavones, namely calycosin and its isomers (F1-F3), calycosin-7-O-glucoside (F4), ononin (F5), formononetin (F6), isomers of pratensein (F7, F8), daidzein (F11), trihydroxyisoflavone/flavone (F13), isomer of odoratin (F14), dihydroxy dimethoxyisoflavone/flavone (F16), naringin (F18), pratensein glucoside and its isomer (F22, F23); four pterocarpans and isoflavanes, namely, astraisoflavane isomer (F15), astraptercarpan (F17), astrapterocarpan pentose glucoside (F20), 3,10-dihydroxy-9-methoxypterocarpan (F21); four dihydroisoflavones/flavones and chalcones, namely trihydroxy-dihydroisoflavone/flavone (F9), trihydroxy-tetrahydroisoflavone/flavone (F10), trihydroxychalcone (F12), dihydrocalycosin pentose glucoside (F19). Among the 23 original constituents, three were newly found original constituents of ARTF, namely F3, F7, and F8. Seventeen original constituents (F1-F17) were found in the urine (Figure 1); F4-F6 (Figure S1) were detected in the plasma; F1, F6, F12, and F17 (Figure S2) existed in the feces. The remaining six original constituents (F18-F23; Table 1) were detected only in the organs.
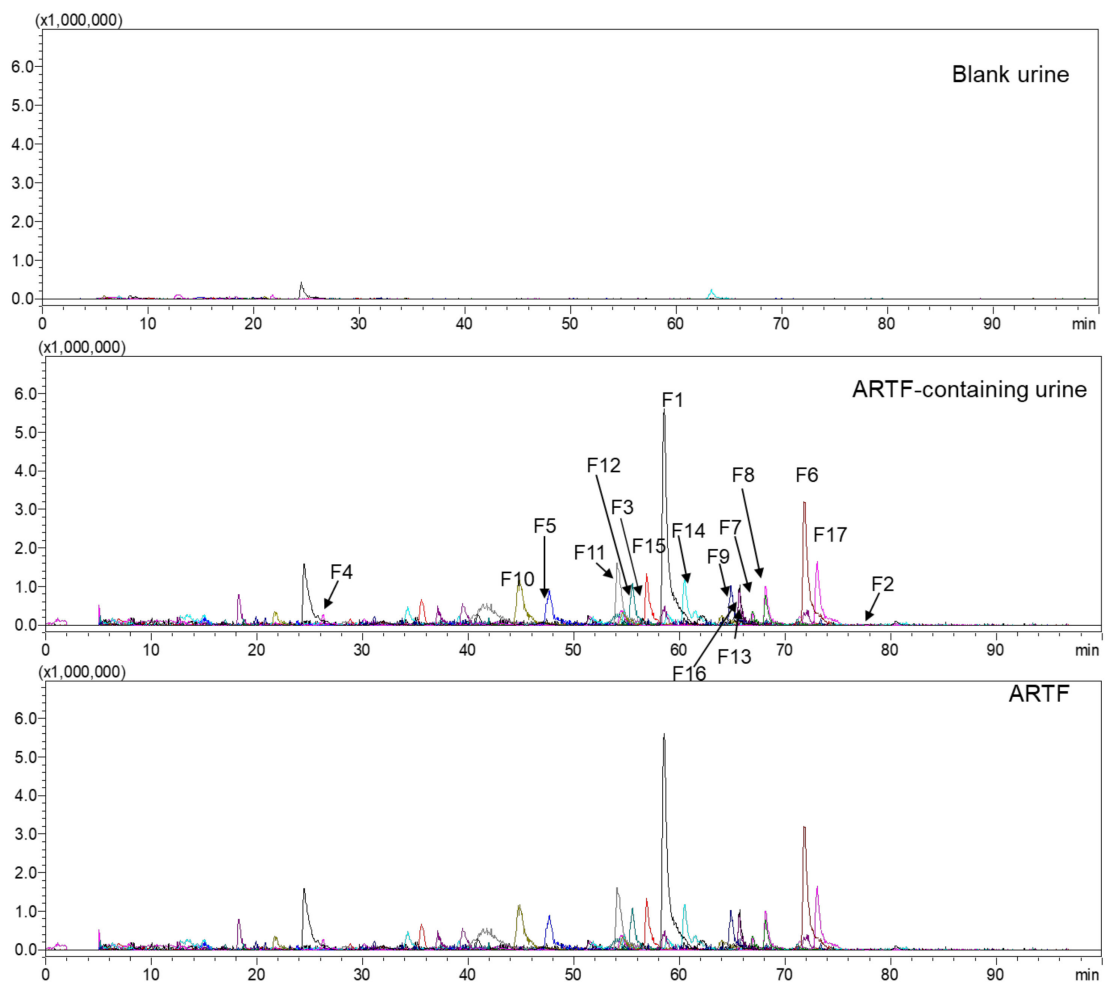

Figure 1. The extracted ion chromatograms (EICs) of original constituents (F1-F17) in rat urine after administration of Astragali Radix total flavonoid (ARTF). 
Table 1. Original constituents in vivo after administration of ARTF to rats

\begin{tabular}{|c|c|c|c|c|c|c|c|c|c|c|c|}
\hline NO. & $\mathbf{t}_{R}(\min )$ & Formula (M) & Ion & $\begin{array}{l}\text { Meas. } \\
(\mathrm{m} / \mathrm{z})\end{array}$ & $\begin{array}{l}\text { Pred. } \\
(\mathrm{m} / \mathrm{z})\end{array}$ & Diff (ppm) & DBE & Identification & Plasma & Urine & Feces \\
\hline $\mathrm{F} 1^{\bullet}$ & 58.602 & $\mathrm{C}_{16} \mathrm{H}_{12} \mathrm{O}_{5}$ & {$[\mathrm{M}-\mathrm{H}]^{-}$} & 283.0623 & 283.0612 & 3.89 & 11 & Calycosin & & $\Delta$ & $\Delta$ \\
\hline F2 & 77.588 & $\mathrm{C}_{16} \mathrm{H}_{12} \mathrm{O}_{5}$ & {$[\mathrm{M}+\mathrm{H}]^{+}$} & 285.0753 & 285.0758 & -1.75 & 11 & Calycosin isomer 1 & & $\Delta$ & \\
\hline F3* & 56.485 & $\mathrm{C}_{16} \mathrm{H}_{12} \mathrm{O}_{5}$ & {$[\mathrm{M}-\mathrm{H}]^{-}$} & 283.0608 & 283.0612 & -1.41 & 11 & Calycosin isomer 2 & & $\Delta$ & \\
\hline $\mathrm{F} 4$ • & 27.200 & $\mathrm{C}_{22} \mathrm{H}_{22} \mathrm{O}_{10}$ & {$\left[\mathrm{M}+\mathrm{HCOO}^{-}\right.$} & 491.1215 & 491.1195 & 4.07 & 12 & Calycosin-7-O-glucoside & $\Delta$ & $\Delta$ & \\
\hline $\mathrm{F} 5$ • & 47.743 & $\mathrm{C}_{22} \mathrm{H}_{22} \mathrm{O}_{9}$ & {$[\mathrm{M}+\mathrm{H}]^{+}$} & 431.1322 & 431.1337 & -3.48 & 12 & Ononin & $\Delta$ & $\Delta$ & \\
\hline $\mathrm{F} 6^{\bullet}$ & 71.750 & $\mathrm{C}_{16} \mathrm{H}_{12} \mathrm{O}_{4}$ & {$[\mathrm{M}-\mathrm{H}]^{-}$} & 267.0673 & 267.0663 & 3.74 & 11 & Formononetin & $\Delta$ & $\Delta$ & $\Delta$ \\
\hline $\mathrm{F} 7$ * & 66.802 & $\mathrm{C}_{16} \mathrm{H}_{12} \mathrm{O}_{6}$ & {$[\mathrm{M}-\mathrm{H}]^{-}$} & 299.0569 & 299.0561 & 2.68 & 11 & Pratensein/Rhamnocitrin/5,7,4'-trihydroxy-3'-methoxyisoflavone & & $\Delta$ & \\
\hline $\mathrm{F} 8$ * & 68.197 & $\mathrm{C}_{16} \mathrm{H}_{12} \mathrm{O}_{6}$ & {$[\mathrm{M}-\mathrm{H}]^{-}$} & 299.0576 & 299.0561 & 5.02 & 11 & Pratensein/Rhamnocitrin/5,7,4'-trihydroxy-3'-methoxyisoflavone & & $\Delta$ & \\
\hline F9 & 65.018 & $\mathrm{C}_{15} \mathrm{H}_{12} \mathrm{O}_{5}$ & {$[\mathrm{M}-\mathrm{H}]^{-}$} & 271.0622 & 271.0612 & 3.69 & 10 & Trihydroxy-dihydroisoflavone/flavone & & $\Delta$ & \\
\hline F10 & 44.750 & $\mathrm{C}_{15} \mathrm{H}_{14} \mathrm{O}_{5}$ & {$[\mathrm{M}-\mathrm{H}]^{-}$} & 273.0777 & 273.0768 & 3.30 & 9 & Trihydroxy-tetrahydroisoflavone/flavone & & $\Delta$ & \\
\hline F11 & 54.377 & $\mathrm{C}_{15} \mathrm{H}_{10} \mathrm{O}_{4}$ & {$[\mathrm{M}-\mathrm{H}]^{-}$} & 253.0514 & 253.0506 & 3.16 & 11 & Daidzein & & $\Delta$ & \\
\hline F12 & 55.485 & $\mathrm{C}_{15} \mathrm{H}_{12} \mathrm{O}_{4}$ & {$[\mathrm{M}-\mathrm{H}]^{-}$} & 255.0675 & 255.0663 & 4.70 & 10 & Trihydroxychalcone & & $\Delta$ & $\Delta$ \\
\hline F13 & 65.627 & $\mathrm{C}_{15} \mathrm{H}_{10} \mathrm{O}_{5}$ & {$[\mathrm{M}-\mathrm{H}]^{-}$} & 269.0452 & 269.0455 & -1.12 & 11 & Trihydroxyisoflavone/flavone & & $\Delta$ & \\
\hline F14 & 60.827 & $\mathrm{C}_{17} \mathrm{H}_{14} \mathrm{O}_{6}$ & {$[\mathrm{M}+\mathrm{H}]^{+}$} & 315.0882 & 315.0863 & 6.03 & 11 & Odoratin isomer & & $\Delta$ & \\
\hline F15 & 57.460 & $\mathrm{C}_{17} \mathrm{H}_{18} \mathrm{O}_{5}$ & {$[\mathrm{M}+\mathrm{H}]^{+}$} & 303.1216 & 303.1227 & -3.63 & 9 & Astraisoflavane isomer & & $\Delta$ & \\
\hline F16 & 65.852 & $\mathrm{C}_{17} \mathrm{H}_{16} \mathrm{O}_{6}$ & {$[\mathrm{M}+\mathrm{H}]^{+}$} & 317.1040 & 317.1020 & 6.31 & 10 & Dihydroxy dimethoxyisoflavone/flavone & & $\Delta$ & \\
\hline $\mathrm{F} 17^{\bullet}$ & 73.328 & $\mathrm{C}_{17} \mathrm{H}_{16} \mathrm{O}_{5}$ & {$[\mathrm{M}+\mathrm{H}]^{+}$} & 301.1052 & 301.1071 & -6.31 & 10 & Astraptercarpan & & $\Delta$ & $\Delta$ \\
\hline F18 & 35.440 & $\mathrm{C}_{27} \mathrm{H}_{32} \mathrm{O}_{14}$ & {$[\mathrm{M}-\mathrm{H}]^{-}$} & 579.1748 & 579.1719 & 5.01 & 12 & Naringin & & & \\
\hline F19 & 34.635 & $\mathrm{C}_{28} \mathrm{H}_{36} \mathrm{O}_{13}$ & {$[\mathrm{M}-\mathrm{H}]^{-}$} & 579.2103 & 579.2083 & 3.45 & 11 & Dihydrocalycosin pentose glucoside & & & \\
\hline F20 & 51.837 & $\mathrm{C}_{28} \mathrm{H}_{34} \mathrm{O}_{14}$ & {$[\mathrm{M}-\mathrm{H}]^{-}$} & 593.1899 & 593.1876 & 3.88 & 12 & Astrapterocarpan pentose glucoside & & & \\
\hline F21 & 63.302 & $\mathrm{C}_{16} \mathrm{H}_{14} \mathrm{O}_{5}$ & {$[\mathrm{M}+\mathrm{H}]^{+}$} & 287.0927 & 287.0914 & 4.53 & 10 & 3,10-dihydroxy-9-methoxypterocarpan & & & \\
\hline F22 & 60.812 & $\mathrm{C}_{22} \mathrm{H}_{22} \mathrm{O}_{11}$ & {$[\mathrm{M}-\mathrm{H}]^{-}$} & 461.1114 & 461.1089 & 5.42 & 12 & $\begin{array}{l}\text { Pratensein glucoside } / 5,7^{\prime}, 4^{\prime} \text {-trihydroxy-3'-methoxyisoflavone } \\
\text { glucoside }\end{array}$ & & & \\
\hline F23 & 22.332 & $\mathrm{C}_{22} \mathrm{H}_{22} \mathrm{O}_{11}$ & {$[\mathrm{M}-\mathrm{H}]^{-}$} & 461.1112 & 461.1089 & 4.99 & 12 & $\begin{array}{c}\begin{array}{c}\text { Pratensein glucoside } / 5,7^{\prime}, 4^{\prime} \text {-trihydroxy-3'-methoxyisoflavone } \\
\text { glucoside }\end{array}\end{array}$ & & & \\
\hline \multicolumn{9}{|r|}{ 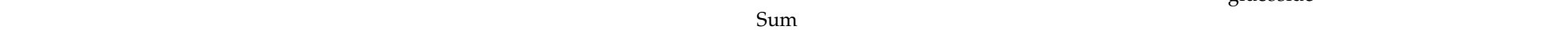 } & 3 & 17 & 4 \\
\hline
\end{tabular}

Note: $t_{R}$ : Retention time; Meas.: measured; Pred.: predicted; Diff: difference; DBE: double bone equivalents. ${ }^{\bullet}$ These constituents were identified by comparison with reference compounds;

* New original constituents found in vivo after administration of ARTF. $\mathbf{\Delta}$ Detected. 


\subsubsection{Identification of Metabolites}

The peaks only appearing in LC-MS chromatograms of ARTF-treated rat bio-samples, but not existing in either blank bio-samples or ARTF were regarded as metabolites. By comparing the EICs and BPCs of them, 147 peaks were assigned as metabolites (M1-M147; Table 2). M145-M147 were only found in the organs. One hundred and six, 64, and 17 metabolites were identified in the urine (Figure 2), plasma (Figure S3), and feces (Figure S4), respectively. Among the 147 metabolites, 89 were new metabolites of ARTF, from which 12 were regarded as new compounds by searching information from SciFinder database (their MS information was shown in Table S1 and Figures S5-S16). Eighty-nine new metabolites included the sulfates of the ring cleavage products of flavone, sulfates of oxidized, reduced, methylated astraisoflavan, and all the glucuronides as well as disulfates. And 12 potential new compounds were sulfates, disulfates, glucuronides, diglucuronides of tetrahydrocalycosin. By analyzing the structures of 147 metabolites, we speculated they were mainly derived from calycosin and its glycoside (maybe the sources of M17-M72, M131-M139), formononetin and its glycosides (maybe the sources of M73-M78, M131-M139), astrapterocarpan-3-O-glucoside (maybe the sources of M79-M84), astraisoflavan-7-O-glucisode (maybe the sources of M85-M106, M147) and many other low content constituents such as astrapterocarpan (maybe one of the sources of M79-M84), astraisoflavan (maybe one of the sources of M85-M106, M147), daidzein (maybe one source of M107-M117, M131-M139), genistein (maybe one source of M118-M130), and so on. Hence, they were speculated to be the main sources of existence forms of ARTF. These metabolites could be classified into 9 phase I metabolites and 138 phase II metabolites. A hundred and thirty-eight phase II metabolites consisted of 7 methyl conjugates, 92 sulfates, 26 glucuronides, and 13 both sulfuric acid and glucuronic acid conjugates, which indicated that sulfates were the most important existence forms of ARTF. The structural elucidation process of some representative metabolites was described as follows.
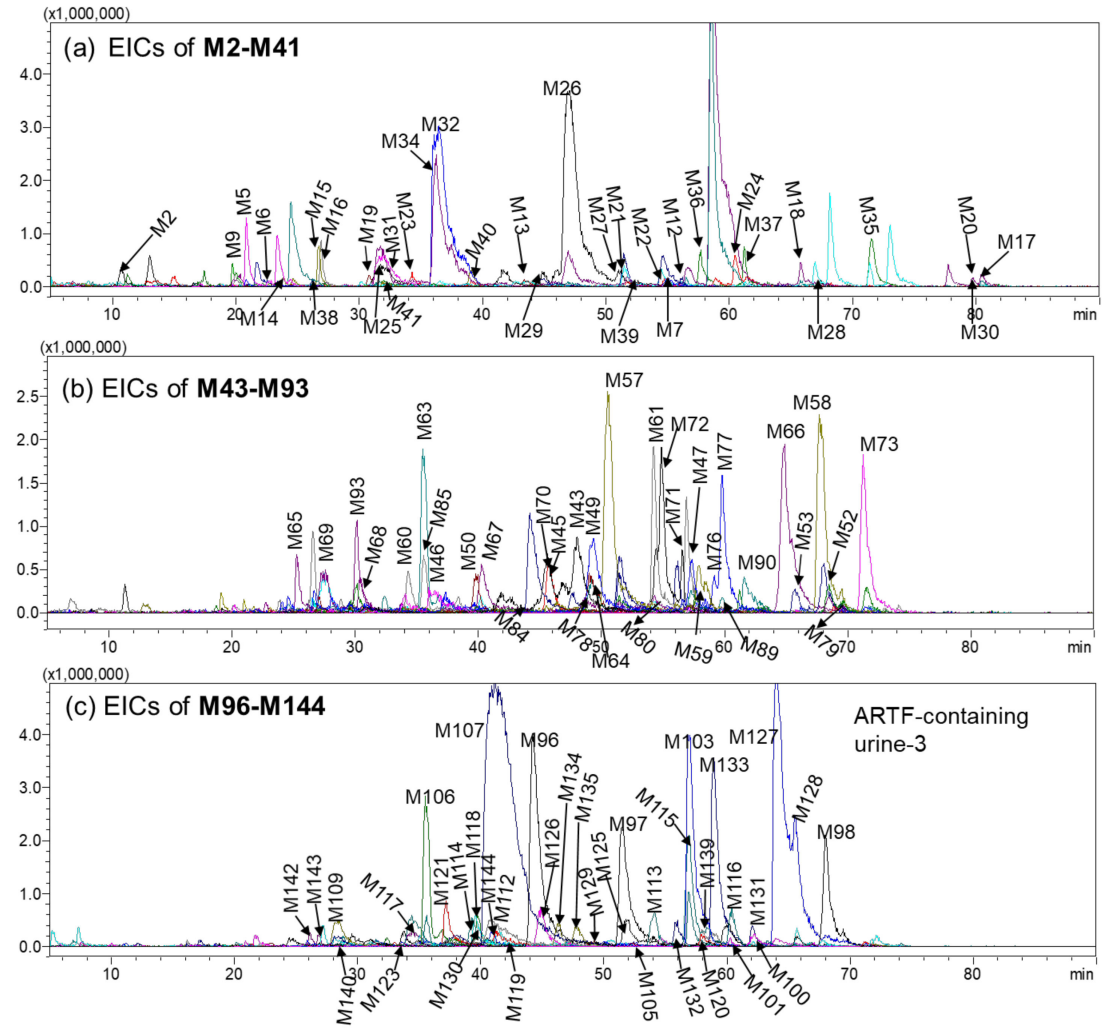

Figure 2. The EICs of 106 metabolites in rat urine after administration of ARTF. (a) EICs of M2-M41; (b) EICs of M43-M93; (c) EICs of M96-M144. 
Table 2. Metabolites in vivo after administration of ARTF to rats.

\begin{tabular}{|c|c|c|c|c|c|c|c|c|c|c|c|}
\hline NO. & $\mathbf{t}_{R}(\min )$ & Formula (M) & Ion & $\begin{array}{l}\text { Meas. } \\
(\mathrm{m} / \mathrm{z})\end{array}$ & $\begin{array}{l}\text { Pred. } \\
(\mathrm{m} / \mathrm{z})\end{array}$ & Diff (ppm) & DBE & Identification & Urine & Plasma & Feces \\
\hline M1 $\triangle$ & 19.500 & $\mathrm{C}_{7} \mathrm{H}_{6} \mathrm{O}_{5} \mathrm{~S}$ & {$[\mathrm{M}-\mathrm{H}]^{-}$} & 200.9862 & 200.9863 & -0.50 & 5 & Hydroxyl-benzaladehyde sulfate or isomer & & $\Delta$ & \\
\hline $\mathrm{M} 2 \triangle$ & 10.948 & $\mathrm{C}_{7} \mathrm{H}_{8} \mathrm{O}_{6} \mathrm{~S}$ & {$[\mathrm{M}-\mathrm{H}]^{-}$} & 218.9967 & 218.9969 & -0.91 & 4 & Methyl pyrogallol sulfate or isomer & $\Delta$ & & \\
\hline M3 ${ }^{\Delta}$ & 20.550 & $\mathrm{C}_{8} \mathrm{H}_{8} \mathrm{O}_{6} \mathrm{~S}$ & {$[\mathrm{M}-\mathrm{H}]^{-}$} & 230.9961 & 230.9969 & -3.46 & 5 & Hydroxyphenylacetic acid sulfate & & $\Delta$ & \\
\hline M4 $\Delta$ & 28.350 & $\mathrm{C}_{8} \mathrm{H}_{8} \mathrm{O}_{6} \mathrm{~S}$ & {$\left[\mathrm{M}-\mathrm{H}^{-}\right.$} & 230.9963 & 230.9969 & -2.6 & 5 & Hydroxyphenylacetic acid sulfate isomer & & $\Delta$ & \\
\hline M5 ${ }^{\Delta}$ & 21.008 & $\mathrm{C}_{9} \mathrm{H}_{8} \mathrm{O}_{6} \mathrm{~S}$ & {$[\mathrm{M}-\mathrm{H}]^{-}$} & 242.9978 & 242.9969 & 3.7 & 6 & Hydroxycinnamic acid sulfate 1 & $\Delta$ & $\Delta$ & \\
\hline 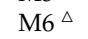 & 23.533 & $\mathrm{C}_{9} \mathrm{H}_{8} \mathrm{O}_{6} \mathrm{~S}$ & {$[\mathrm{M}-\mathrm{H}]^{-}$} & 242.9960 & 242.9969 & -3.70 & 6 & Hydroxycinnamic acid sulfate 2 & $\Delta$ & & \\
\hline $\mathrm{M}^{\triangle} \triangle$ & 55.018 & $\mathrm{C}_{10} \mathrm{H}_{12} \mathrm{O}_{5} \mathrm{~S}$ & {$[\mathrm{M}-\mathrm{H}]^{-}$} & 243.0345 & 243.0333 & 4.94 & 5 & Eugenol sulfate & $\Delta$ & & \\
\hline $\mathrm{M}^{\Delta}$ & 36.100 & $\mathrm{C}_{8} \mathrm{H}_{8} \mathrm{O}_{7} \mathrm{~S}$ & {$[\mathrm{M}-\mathrm{H}]^{-}$} & 246.9921 & 246.9918 & 1.21 & 5 & Vanillic acid sulfate & & $\Delta$ & \\
\hline M9 $\triangle$ & 19.617 & $\mathrm{C}_{9} \mathrm{H}_{12} \mathrm{O}_{6} \mathrm{~S}$ & {$[\mathrm{M}-\mathrm{H}]^{-}$} & 247.0281 & 247.0282 & -0.40 & 4 & Homovanillyl alcohol sulfate & $\Delta$ & & \\
\hline $\mathrm{M} 10^{\Delta}$ & 20.383 & $\mathrm{C}_{10} \mathrm{H}_{10} \mathrm{O}_{7} \mathrm{~S}$ & {$[\mathrm{M}-\mathrm{H}]^{-}$} & 273.0080 & 273.0074 & 2.20 & 6 & Ferulic Acid sulfate & & $\Delta$ & \\
\hline M11 $\Delta$ & 58.818 & $\mathrm{C}_{11} \mathrm{H}_{14} \mathrm{O}_{6} \mathrm{~S}$ & {$[\mathrm{M}-\mathrm{H}]^{-}$} & 273.0436 & 273.0438 & -0.73 & 5 & Methoxyeugenol sulfate 1 & & $\Delta$ & \\
\hline $\mathrm{M} 12^{\Delta}$ & 55.593 & $\mathrm{C}_{11} \mathrm{H}_{14} \mathrm{O}_{6} \mathrm{~S}$ & {$[\mathrm{M}-\mathrm{H}]^{-}$} & 273.0433 & 273.0438 & -1.83 & 5 & Methoxyeugenol sulfate 2 & $\Delta$ & $\Delta$ & \\
\hline M13 $\Delta$ & 43.617 & $\mathrm{C}_{11} \mathrm{H}_{12} \mathrm{O}_{7} \mathrm{~S}$ & {$[\mathrm{M}-\mathrm{H}]^{-}$} & 287.0237 & 287.0231 & 2.09 & 6 & $\mathrm{C}_{11} \mathrm{H}_{12} \mathrm{O}_{4}$ sulfate & $\Delta$ & & \\
\hline M14 ${ }^{\Delta}$ & 23.750 & $\mathrm{C}_{11} \mathrm{H}_{14} \mathrm{O}_{7} \mathrm{~S}$ & {$[\mathrm{M}-\mathrm{H}]^{-}$} & 289.0393 & 289.0387 & 2.08 & 5 & Ethylhomovanillic acid sulfate 1 & $\Delta$ & $\Delta$ & \\
\hline M15 $\Delta$ & 26.642 & $\mathrm{C}_{11} \mathrm{H}_{14} \mathrm{O}_{7} \mathrm{~S}$ & {$[\mathrm{M}-\mathrm{H}]^{-}$} & 289.0397 & 289.0387 & 3.46 & 5 & Ethylhomovanillic acid sulfate 2 & $\Delta$ & $\Delta$ & \\
\hline $\mathrm{M} 16^{\Delta}$ & 27.558 & $\mathrm{C}_{11} \mathrm{H}_{12} \mathrm{O}_{8} \mathrm{~S}$ & {$[\mathrm{M}-\mathrm{H}]^{-}$} & 303.0173 & 303.018 & -2.31 & 6 & $3^{\prime}, 5^{\prime}$-dimethoxy-4'-hydroxycinnamic acid sulfate & $\Delta$ & $\Delta$ & \\
\hline M17 & 80.230 & $\mathrm{C}_{16} \mathrm{H}_{12} \mathrm{O}_{5}$ & {$[\mathrm{M}-\mathrm{H}]^{-}$} & 283.0604 & 283.0612 & -2.83 & 11 & Calycosin isomer 1 & $\Delta$ & & \\
\hline${\mathrm{M} 18^{\Delta}}^{\Delta}$ & 65.833 & $\mathrm{C}_{16} \mathrm{H}_{12} \mathrm{O}_{5}$ & {$[\mathrm{M}+\mathrm{H}]^{+}$} & 285.0767 & 285.0758 & 3.16 & 11 & Calycosin isomer 2 & $\Delta$ & & \\
\hline M19 $\Delta$ & 31.508 & $\mathrm{C}_{16} \mathrm{H}_{12} \mathrm{O}_{5}$ & {$[\mathrm{M}+\mathrm{H}]^{+}$} & 285.0738 & 285.0758 & -7.02 & 11 & Calycosin isomer 3 & $\Delta$ & $\Delta$ & \\
\hline $\mathrm{M} 20^{\Delta}$ & 79.592 & $\mathrm{C}_{16} \mathrm{H}_{12} \mathrm{O}_{5}$ & {$[\mathrm{M}+\mathrm{H}]^{+}$} & 285.0777 & 285.0758 & 6.66 & 11 & Calycosin isomer 4 & $\Delta$ & & \\
\hline M21 & 51.610 & $\mathrm{C}_{16} \mathrm{H}_{12} \mathrm{O}_{6}$ & {$[\mathrm{M}+\mathrm{H}]^{+}$} & 301.0688 & 301.0707 & -6.31 & 11 & Hydroxycalycosin & $\Delta$ & & \\
\hline M22 & 54.902 & $\mathrm{C}_{17} \mathrm{H}_{14} \mathrm{O}_{6}$ & {$[\mathrm{M}+\mathrm{H}]^{+}$} & 315.0883 & 315.0863 & 6.35 & 11 & Methoxycalycosin 1 & $\Delta$ & & \\
\hline M23 & 34.517 & $\mathrm{C}_{17} \mathrm{H}_{14} \mathrm{O}_{6}$ & {$[\mathrm{M}+\mathrm{H}]^{+}$} & 315.0836 & 315.0863 & -8.57 & 11 & Methoxycalycosin 2 & $\Delta$ & & \\
\hline M24 & 61.477 & $\mathrm{C}_{17} \mathrm{H}_{14} \mathrm{O}_{6}$ & {$[\mathrm{M}+\mathrm{H}]^{+}$} & 315.0840 & 315.0863 & -7.3 & 11 & Methoxycalycosin 3 & $\Delta$ & & \\
\hline M25 & 31.483 & $\mathrm{C}_{16} \mathrm{H}_{12} \mathrm{O}_{8} \mathrm{~S}$ & {$[\mathrm{M}-\mathrm{H}]^{-}$} & 363.0178 & 363.0180 & -0.55 & 11 & Calycosin sulfate 1 & $\Delta$ & $\Delta$ & \\
\hline M26 & 47.512 & $\mathrm{C}_{16} \mathrm{H}_{12} \mathrm{O}_{8} \mathrm{~S}$ & {$[\mathrm{M}-\mathrm{H}]^{-}$} & 363.018 & 363.0180 & 0 & 11 & Calycosin sulfate 2 & $\Delta$ & $\Delta$ & \\
\hline M27 & 51.168 & $\mathrm{C}_{16} \mathrm{H}_{12} \mathrm{O}_{8} \mathrm{~S}$ & {$[\mathrm{M}-\mathrm{H}]^{-}$} & 363.0187 & 363.0180 & 1.93 & 11 & Calycosin sulfate isomer 1 & $\Delta$ & $\Delta$ & \\
\hline $\mathrm{M} 28^{\Delta}$ & 67.248 & $\mathrm{C}_{16} \mathrm{H}_{12} \mathrm{O}_{8} \mathrm{~S}$ & {$[\mathrm{M}-\mathrm{H}]^{-}$} & 363.0174 & 363.0180 & -1.65 & 11 & Calycosin sulfate isomer 2 & $\Delta$ & & \\
\hline $\mathrm{M} 29^{\Delta}$ & 44.867 & $\mathrm{C}_{16} \mathrm{H}_{12} \mathrm{O}_{8} \mathrm{~S}$ & {$[\mathrm{M}-\mathrm{H}]^{-}$} & 363.0189 & 363.0180 & 2.48 & 11 & Calycosin sulfate isomer 3 & $\Delta$ & $\Delta$ & \\
\hline $\mathrm{M} 30^{\Delta}$ & 79.697 & $\mathrm{C}_{16} \mathrm{H}_{12} \mathrm{O}_{8} \mathrm{~S}$ & {$[\mathrm{M}-\mathrm{H}]^{-}$} & 363.0193 & 363.0180 & 3.58 & 11 & Calycosin sulfate isomer 4 & $\Delta$ & & \\
\hline $\mathrm{M} 31^{\Delta}$ & 33.158 & $\mathrm{C}_{16} \mathrm{H}_{12} \mathrm{O}_{11} \mathrm{~S}_{2}$ & {$[\mathrm{M}-\mathrm{H}]^{-}$} & 442.9767 & 442.9748 & 4.29 & 11 & Calycosin- $7,3^{\prime}-O$-disulfate & $\Delta$ & $\Delta$ & \\
\hline $\mathrm{M} 32^{\Delta}$ & 36.133 & $\mathrm{C}_{22} \mathrm{H}_{20} \mathrm{O}_{11}$ & {$[\mathrm{M}-\mathrm{H}]^{-}$} & 459.0952 & 459.0933 & 4.14 & 13 & Calycosin-3'-O-glucuronide & $\Delta$ & $\Delta$ & \\
\hline $\mathrm{M} 33^{\Delta}$ & 23.417 & $\mathrm{C}_{22} \mathrm{H}_{20} \mathrm{O}_{14} \mathrm{~S}$ & {$[\mathrm{M}-\mathrm{H}]^{-}$} & 539.0538 & 539.0501 & 6.86 & 13 & Calycosin glucuronide sulfate 1 & & $\Delta$ & \\
\hline $\mathrm{M} 34^{\Delta}$ & 31.245 & $\mathrm{C}_{22} \mathrm{H}_{20} \mathrm{O}_{14} \mathrm{~S}$ & {$[\mathrm{M}-\mathrm{H}]^{-}$} & 539.0519 & 539.0501 & 3.34 & 13 & Calycosin glucuronide sulfate 2 & $\Delta$ & $\Delta$ & \\
\hline M35 & 71.298 & $\mathrm{C}_{16} \mathrm{H}_{12} \mathrm{O}_{9} \mathrm{~S}$ & {$[\mathrm{M}-\mathrm{H}]^{-}$} & 379.0137 & 379.0129 & 2.11 & 11 & Hydroxycalycosin sulfate 1 & $\Delta$ & $\Delta$ & \\
\hline M36 & 57.743 & $\mathrm{C}_{16} \mathrm{H}_{12} \mathrm{O}_{9} \mathrm{~S}$ & {$[\mathrm{M}-\mathrm{H}]^{-}$} & 379.0153 & 379.0129 & 6.33 & 11 & Hydroxycalycosin sulfate 2 & $\Delta$ & & \\
\hline M37 & 61.243 & $\mathrm{C}_{16} \mathrm{H}_{12} \mathrm{O}_{9} \mathrm{~S}$ & {$[\mathrm{M}-\mathrm{H}]^{-}$} & 379.0151 & 379.0129 & 5.80 & 11 & Hydroxycalycosin sulfate 3 & $\Delta$ & & \\
\hline $\mathrm{M} 38^{\Delta}$ & 26.125 & $\mathrm{C}_{22} \mathrm{H}_{20} \mathrm{O}_{12}$ & {$[\mathrm{M}-\mathrm{H}]^{-}$} & 475.0847 & 475.0882 & -7.37 & 13 & Hydroxycalycosin glucuronide 1 & $\Delta$ & & \\
\hline M39 $\Delta$ & 51.610 & $\mathrm{C}_{22} \mathrm{H}_{20} \mathrm{O}_{12}$ & {$[\mathrm{M}-\mathrm{H}]^{-}$} & 475.0918 & 475.0882 & 7.58 & 13 & Hydroxycalycosin glucuronide 2 & $\Delta$ & & \\
\hline $\mathrm{M} 40^{\Delta}$ & 39.042 & $\mathrm{C}_{23} \mathrm{H}_{22} \mathrm{O}_{12}$ & {$[\mathrm{M}-\mathrm{H}]^{-}$} & 489.1063 & 489.1038 & 5.11 & 13 & Methoxycalycosin glucuronide & $\Delta$ & $\Delta$ & \\
\hline $\mathrm{M} 41^{\Delta}$ & 33.123 & $\mathrm{C}_{23} \mathrm{H}_{22} \mathrm{O}_{15} \mathrm{~S}$ & {$[\mathrm{M}-\mathrm{H}]^{-}$} & 569.0634 & 569.0607 & 4.74 & 13 & Methoxycalycosin glucuronide sulfate & $\Delta$ & $\Delta$ & \\
\hline $\mathrm{M} 42^{\Delta}$ & 20.108 & $\mathrm{C}_{28} \mathrm{H}_{30} \mathrm{O}_{16}$ & {$[\mathrm{M}-\mathrm{H}]^{-}$} & 621.1451 & 621.1461 & -1.61 & 14 & Calycosin-7-O-glucoside-3'-O-glucuronide & & $\Delta$ & \\
\hline M43 & 48.258 & $\mathrm{C}_{16} \mathrm{H}_{14} \mathrm{O}_{8} \mathrm{~S}$ & {$[\mathrm{M}-\mathrm{H}]^{-}$} & 365.0341 & 365.0337 & 1.1 & 10 & Dihydrocalycosin sulfate 1 & $\Delta$ & & \\
\hline
\end{tabular}


Table 2. Cont

\begin{tabular}{|c|c|c|c|c|c|c|c|c|c|c|c|}
\hline NO. & $\mathbf{t}_{R}(\min )$ & Formula (M) & Ion & $\begin{array}{l}\text { Meas. } \\
(m / z)\end{array}$ & $\begin{array}{l}\text { Pred. } \\
(\mathrm{m} / \mathrm{z})\end{array}$ & Diff (ppm) & DBE & Identification & Urine & Plasma & Feces \\
\hline M44 & 43.285 & $\mathrm{C}_{16} \mathrm{H}_{14} \mathrm{O}_{8} \mathrm{~S}$ & {$[\mathrm{M}-\mathrm{H}]^{-}$} & 365.0346 & 365.0337 & 2.47 & 10 & Dihydrocalycosin sulfate 2 & & $\Delta$ & \\
\hline M45 & 45.652 & $\mathrm{C}_{16} \mathrm{H}_{14} \mathrm{O}_{8} \mathrm{~S}$ & {$[\mathrm{M}-\mathrm{H}]^{-}$} & 365.0356 & 365.0337 & 5.2 & 10 & Dihydrocalycosin sulfate 3 & $\Delta$ & $\Delta$ & \\
\hline $\mathrm{M}_{4}{ }^{\Delta}$ & 38.098 & $\mathrm{C}_{22} \mathrm{H}_{22} \mathrm{O}_{11}$ & {$[\mathrm{M}-\mathrm{H}]^{-}$} & 461.1111 & 461.1089 & 4.77 & 12 & Dihydrocalycosin glucuronide & $\Delta$ & & \\
\hline M47 & 57.285 & $\mathrm{C}_{16} \mathrm{H}_{14} \mathrm{O}_{9} \mathrm{~S}$ & {$[\mathrm{M}-\mathrm{H}]^{-}$} & 381.0282 & 381.0286 & -1.05 & 10 & Hydroxy dihydrocalycosin sulfate 1 & $\Delta$ & $\Delta$ & \\
\hline M48 & 93.980 & $\mathrm{C}_{16} \mathrm{H}_{14} \mathrm{O}_{9} \mathrm{~S}$ & {$[\mathrm{M}-\mathrm{H}]^{-}$} & 381.0296 & 381.0286 & 2.62 & 10 & Hydroxy dihydrocalycosin sulfate 2 & $\Delta$ & & \\
\hline M49 & 49.422 & $\mathrm{C}_{16} \mathrm{H}_{14} \mathrm{O}_{9} \mathrm{~S}$ & {$[\mathrm{M}-\mathrm{H}]^{-}$} & 381.0300 & 381.0286 & 3.67 & 10 & Hydroxy dihydrocalycosin sulfate 3 & $\Delta$ & $\Delta$ & \\
\hline M50 & 39.792 & $\mathrm{C}_{18} \mathrm{H}_{18} \mathrm{O}_{8} \mathrm{~S}$ & {$[\mathrm{M}-\mathrm{H}]^{-}$} & 393.0665 & 393.065 & 3.82 & 10 & Dimethyl dihydrocalycosin sulfate & $\Delta$ & & \\
\hline M51 $\Delta$ & 52.118 & $\mathrm{C}_{17} \mathrm{H}_{16} \mathrm{O}_{9} \mathrm{~S}$ & {$[\mathrm{M}-\mathrm{H}]^{-}$} & 395.0434 & 395.0442 & -2.03 & 10 & Methoxy dihydrocalycosin sulfate 1 & & $\Delta$ & $\Delta$ \\
\hline $\mathrm{M} 52^{\Delta}$ & 68.430 & $\mathrm{C}_{17} \mathrm{H}_{16} \mathrm{O}_{9} \mathrm{~S}$ & {$[\mathrm{M}-\mathrm{H}]^{-}$} & 395.0457 & 395.0442 & 3.80 & 10 & Methoxy dihydrocalycosin sulfate 2 & $\Delta$ & & \\
\hline M53 & 66.472 & $\mathrm{C}_{16} \mathrm{H}_{14} \mathrm{O}_{10} \mathrm{~S}$ & {$[\mathrm{M}-\mathrm{H}]^{-}$} & 397.0251 & 397.0235 & 4.03 & 10 & Dihydroxyl dihydrocalycosin sulfate & $\Delta$ & & \\
\hline M54 & 25.252 & $\mathrm{C}_{16} \mathrm{H}_{16} \mathrm{O}_{8} \mathrm{~S}$ & {$[\mathrm{M}-\mathrm{H}]^{-}$} & 367.0477 & 367.0493 & -4.36 & 9 & Tetrahydrocalycosin sulfate 1 & & & $\Delta$ \\
\hline M55 & 19.342 & $\mathrm{C}_{16} \mathrm{H}_{16} \mathrm{O}_{8} \mathrm{~S}$ & {$[\mathrm{M}-\mathrm{H}]^{-}$} & 367.0496 & 367.0493 & 0.82 & 9 & Tetrahydrocalycosin sulfate 2 & & & $\Delta$ \\
\hline M56 & 21.443 & $\mathrm{C}_{16} \mathrm{H}_{16} \mathrm{O}_{8} \mathrm{~S}$ & {$[\mathrm{M}-\mathrm{H}]^{-}$} & 367.0500 & 367.0493 & 1.91 & 9 & Tetrahydrocalycosin sulfate 3 & & & $\Delta$ \\
\hline M57 & 50.593 & $\mathrm{C}_{16} \mathrm{H}_{16} \mathrm{O}_{8} \mathrm{~S}$ & {$[\mathrm{M}-\mathrm{H}]^{-}$} & 367.0516 & 367.0493 & 6.27 & 9 & Tetrahydrocalycosin sulfate 4 & $\Delta$ & $\Delta$ & \\
\hline M58 & 67.630 & $\mathrm{C}_{16} \mathrm{H}_{16} \mathrm{O}_{8} \mathrm{~S}$ & {$[\mathrm{M}-\mathrm{H}]^{-}$} & 367.0517 & 367.0493 & 6.54 & 9 & Tetrahydrocalycosin sulfate 5 & $\Delta$ & $\Delta$ & \\
\hline M59 & 57.860 & $\mathrm{C}_{16} \mathrm{H}_{16} \mathrm{O}_{8} \mathrm{~S}$ & {$[\mathrm{M}-\mathrm{H}]^{-}$} & 367.0518 & 367.0493 & 6.81 & 9 & Tetrahydrocalycosin sulfate 6 & $\Delta$ & & \\
\hline $\mathrm{M} 60^{\Delta}$ & 34.182 & $\mathrm{C}_{22} \mathrm{H}_{24} \mathrm{O}_{11}$ & {$[\mathrm{M}-\mathrm{H}]^{-}$} & 463.1257 & 463.1246 & 2.38 & 11 & Tetrahydrocalycosin glucuronide 1 & $\Delta$ & $\Delta$ & \\
\hline M61 $\triangle$ & 54.027 & $\mathrm{C}_{22} \mathrm{H}_{24} \mathrm{O}_{11}$ & {$[\mathrm{M}-\mathrm{H}]^{-}$} & 463.1269 & 463.1246 & 4.97 & 11 & Tetrahydrocalycosin glucuronide 2 & $\Delta$ & $\Delta$ & \\
\hline $\mathrm{M} 62^{\triangle, \star}$ & 26.975 & $\mathrm{C}_{22} \mathrm{H}_{24} \mathrm{O}_{14} \mathrm{~S}$ & {$[\mathrm{M}-\mathrm{H}]^{-}$} & 543.0792 & 543.0814 & -4.05 & 11 & Tetrahydrocalycosin glucuronide sulfate & & $\Delta$ & \\
\hline $\mathrm{M} 63^{\Delta, \star}$ & 32.350 & $\mathrm{C}_{28} \mathrm{H}_{32} \mathrm{O}_{17}$ & {$[\mathrm{M}-\mathrm{H}]^{-}$} & 639.1607 & 639.1567 & 6.26 & 13 & Tetrahydrocalycosin diglucuronide & $\Delta$ & & \\
\hline M64 & 48.375 & $\mathrm{C}_{16} \mathrm{H}_{16} \mathrm{O}_{9} \mathrm{~S}$ & {$[\mathrm{M}-\mathrm{H}]^{-}$} & 383.0449 & 383.0442 & 1.83 & 9 & Hydroxy tetrahydrocalycosin sulfate 1 & $\Delta$ & & \\
\hline M65 & 25.200 & $\mathrm{C}_{16} \mathrm{H}_{16} \mathrm{O}_{9} \mathrm{~S}$ & {$[\mathrm{M}-\mathrm{H}]^{-}$} & 383.046 & 383.0442 & 4.70 & 9 & Hydroxy tetrahydrocalycosin sulfate 2 & $\Delta$ & & $\Delta$ \\
\hline M66 & 64.843 & $\mathrm{C}_{16} \mathrm{H}_{16} \mathrm{O}_{9} \mathrm{~S}$ & {$[\mathrm{M}-\mathrm{H}]^{-}$} & 383.0462 & 383.0442 & 5.22 & 9 & Hydroxy tetrahydrocalycosin sulfate 3 & $\Delta$ & & \\
\hline M67 & 40.192 & $\mathrm{C}_{16} \mathrm{H}_{16} \mathrm{O}_{9} \mathrm{~S}$ & {$[\mathrm{M}-\mathrm{H}]^{-}$} & 383.0467 & 383.0442 & 6.53 & 9 & Hydroxy tetrahydrocalycosin sulfate 4 & $\Delta$ & & \\
\hline M68 & 30.608 & $\mathrm{C}_{16} \mathrm{H}_{16} \mathrm{O}_{9} \mathrm{~S}$ & {$[\mathrm{M}-\mathrm{H}]^{-}$} & 383.0444 & 383.0442 & 0.52 & 9 & Hydroxy tetrahydrocalycosin sulfate 5 & $\Delta$ & & \\
\hline M69 $\Delta, \star$ & 27.617 & $\mathrm{C}_{16} \mathrm{H}_{16} \mathrm{O}_{12} \mathrm{~S}_{2}$ & {$[\mathrm{M}-\mathrm{H}]^{-}$} & 463.0031 & 463.0010 & 4.75 & 9 & Hydroxy tetrahydrocalycosin disulfate & $\Delta$ & & \\
\hline M70 $\Delta, \star$ & 45.733 & $\mathrm{C}_{18} \mathrm{H}_{20} \mathrm{O}_{10} \mathrm{~S}$ & {$[\mathrm{M}-\mathrm{H}]^{-}$} & 427.0716 & 427.0704 & 2.81 & 9 & Dihydroxy dihydrocalycosin sulfate & $\Delta$ & & \\
\hline $\mathrm{M} 71^{\triangle}$ & 56.593 & $\mathrm{C}_{24} \mathrm{H}_{28} \mathrm{O}_{12}$ & {$[\mathrm{M}-\mathrm{H}]^{-}$} & 507.1535 & 507.1508 & 5.32 & 11 & $\begin{array}{l}\text { Dimethyl hydroxy tetrahydrocalycosin } \\
\text { glucuronide } 1\end{array}$ & $\Delta$ & $\Delta$ & \\
\hline $\mathrm{M} 72 \triangle^{\triangle}$ & 54.727 & $\mathrm{C}_{24} \mathrm{H}_{28} \mathrm{O}_{12}$ & {$[\mathrm{M}-\mathrm{H}]^{-}$} & 507.1539 & 507.1508 & 6.11 & 11 & $\begin{array}{l}\text { Dimethyl hydroxy tetrahydrocalycosin } \\
\text { glucuronide } 2\end{array}$ & $\Delta$ & $\Delta$ & \\
\hline 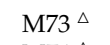 & 71.300 & $\mathrm{C}_{16} \mathrm{H}_{12} \mathrm{O}_{7} \mathrm{~S}$ & {$[\mathrm{M}-\mathrm{H}]^{-}$} & 347.0228 & 347.0231 & -0.86 & 11 & Formononetin-7-O-sulfate & $\Delta$ & $\Delta$ & \\
\hline${\mathrm{M} 74^{\triangle}}$ & 49.357 & $\mathrm{C}_{22} \mathrm{H}_{20} \mathrm{O}_{10}$ & {$[\mathrm{M}-\mathrm{H}]^{-}$} & 443.0977 & 443.0984 & -1.58 & 13 & Formononetin-7-O-glucuronide & & $\Delta$ & \\
\hline M75 & 21.960 & $\mathrm{C}_{16} \mathrm{H}_{16} \mathrm{O}_{7} \mathrm{~S}$ & {$[\mathrm{M}-\mathrm{H}]^{-}$} & 351.0537 & 351.0544 & -1.99 & 9 & Tetrahydroformononetin sulfate 1 & & & $\Delta$ \\
\hline M76 & 59.352 & $\mathrm{C}_{16} \mathrm{H}_{16} \mathrm{O}_{7} \mathrm{~S}$ & {$[\mathrm{M}-\mathrm{H}]^{-}$} & 351.0554 & 351.0544 & 2.85 & 9 & Tetrahydroformononetin sulfate 2 & $\Delta$ & $\Delta$ & \\
\hline M77 & 59.860 & $\mathrm{C}_{16} \mathrm{H}_{16} \mathrm{O}_{7} \mathrm{~S}$ & {$[\mathrm{M}-\mathrm{H}]^{-}$} & 351.0564 & 351.0544 & 5.70 & 9 & Tetrahydroformononetin sulfate 3 & $\Delta$ & & \\
\hline${\mathrm{M} 78^{\Delta}}$ & 49.257 & $\mathrm{C}_{22} \mathrm{H}_{24} \mathrm{O}_{10}$ & {$[\mathrm{M}-\mathrm{H}]^{-}$} & 447.1322 & 447.1297 & 5.59 & 11 & Tetrahydroformononetin glucuronide & $\Delta$ & $\Delta$ & \\
\hline $\mathrm{M} 79 \triangle^{\Delta}$ & 69.797 & $\mathrm{C}_{17} \mathrm{H}_{16} \mathrm{O}_{8} \mathrm{~S}$ & {$[\mathrm{M}-\mathrm{H}]^{-}$} & 379.0509 & 379.0493 & 4.22 & 10 & Astrapterocarpan-3-O-sulfate & $\Delta$ & & \\
\hline $\mathrm{M} 80^{\Delta}$ & 56.043 & $\mathrm{C}_{23} \mathrm{H}_{24} \mathrm{O}_{11}$ & {$[\mathrm{M}-\mathrm{H}]^{-}$} & 475.1276 & 475.1246 & 6.31 & 12 & Astrapterocarpan-3-O-glucuronide & $\Delta$ & $\Delta$ & \\
\hline M81 & 31.018 & $\mathrm{C}_{18} \mathrm{H}_{18} \mathrm{O}_{6}$ & {$[\mathrm{M}-\mathrm{H}]^{-}$} & 329.1027 & 329.1031 & -1.22 & 10 & Methoxyastrapterocarpan & & & $\Delta$ \\
\hline $\mathrm{M} 82^{\Delta}$ & 53.593 & $\mathrm{C}_{23} \mathrm{H}_{24} \mathrm{O}_{12}$ & {$[\mathrm{M}-\mathrm{H}]^{-}$} & 491.1225 & 491.1195 & 6.11 & 12 & Hydroxyastrapterocarpan glucuronide 1 & & $\Delta$ & \\
\hline${\mathrm{M} 83^{\Delta}}$ & 40.915 & $\mathrm{C}_{23} \mathrm{H}_{24} \mathrm{O}_{12}$ & {$[\mathrm{M}-\mathrm{H}]^{-}$} & 491.1194 & 491.1195 & -0.20 & 12 & Hydroxyastrapterocarpan glucuronide 2 & & $\Delta$ & \\
\hline $\mathrm{M} 84^{\triangle}$ & 43.618 & $\mathrm{C}_{23} \mathrm{H}_{24} \mathrm{O}_{12}$ & {$[\mathrm{M}-\mathrm{H}]^{-}$} & 491.1173 & 491.1195 & -4.48 & 12 & Hydroxyastrapterocarpan glucuronide 3 & $\Delta$ & & \\
\hline
\end{tabular}


Table 2. Cont

\begin{tabular}{|c|c|c|c|c|c|c|c|c|c|c|c|}
\hline NO. & $\mathbf{t}_{R}(\min )$ & Formula (M) & Ion & $\begin{array}{l}\text { Meas. } \\
(\mathrm{m} / \mathrm{z})\end{array}$ & $\begin{array}{l}\text { Pred. } \\
(\mathrm{m} / \mathrm{z})\end{array}$ & Diff (ppm) & DBE & Identification & Urine & Plasma & Fece: \\
\hline 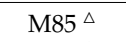 & 35.667 & $\mathrm{C}_{17} \mathrm{H}_{18} \mathrm{O}_{5}$ & {$[\mathrm{M}+\mathrm{H}]^{+}$} & 303.1205 & 303.1227 & -7.26 & 9 & Astraisoflavan isomer & $\Delta$ & & \\
\hline $\mathrm{M}_{8}{ }^{\Delta}$ & 32.868 & $\mathrm{C}_{18} \mathrm{H}_{20} \mathrm{O}_{5}$ & {$[\mathrm{M}-\mathrm{H}]^{-}$} & 315.1228 & 315.1238 & -3.17 & 9 & Methoxyastraisoflavan & & & $\Delta$ \\
\hline $\mathrm{M} 87^{\Delta}$ & 37.802 & $\mathrm{C}_{18} \mathrm{H}_{20} \mathrm{O}_{5}$ & {$[\mathrm{M}-\mathrm{H}]^{-}$} & 315.1235 & 315.1238 & -0.95 & 9 & Methoxyastraisoflavan isomer & & & $\Delta$ \\
\hline $\mathrm{M} 88^{\Delta}$ & 34.368 & $\mathrm{C}_{19} \mathrm{H}_{22} \mathrm{O}_{6}$ & {$[\mathrm{M}-\mathrm{H}]^{-}$} & 345.1360 & 345.1344 & 4.64 & 9 & Hydroxy dimethoxyastraisoflavan & & & $\Delta$ \\
\hline M89 & 59.918 & $\mathrm{C}_{17} \mathrm{H}_{18} \mathrm{O}_{8} \mathrm{~S}$ & {$[\mathrm{M}-\mathrm{H}]^{-}$} & 381.0639 & 381.0650 & -2.89 & 9 & Astraisoflavan-7-O-sulfate & $\Delta$ & & \\
\hline M90 & 62.510 & $\mathrm{C}_{17} \mathrm{H}_{18} \mathrm{O}_{8} \mathrm{~S}$ & {$[\mathrm{M}-\mathrm{H}]^{-}$} & 381.0660 & 381.0650 & 2.62 & 9 & Astraisoflavan-2'-O-sulfate & $\Delta$ & $\Delta$ & \\
\hline M91 & 34.427 & $\mathrm{C}_{17} \mathrm{H}_{18} \mathrm{O}_{8} \mathrm{~S}$ & {$[\mathrm{M}-\mathrm{H}]^{-}$} & 381.0662 & 381.0650 & 3.15 & 9 & Astraisoflavan sulfate isomer & & & $\Delta$ \\
\hline $\mathrm{M} 92^{\triangle}$ & 34.993 & $\mathrm{C}_{18} \mathrm{H}_{20} \mathrm{O}_{8} \mathrm{~S}$ & {$[\mathrm{M}-\mathrm{H}]^{-}$} & 395.0795 & 395.0806 & -2.78 & 9 & Methyoxyastraisoflavan sulfate 1 & & & $\Delta$ \\
\hline M93 $\triangle$ & 30.460 & $\mathrm{C}_{18} \mathrm{H}_{20} \mathrm{O}_{8} \mathrm{~S}$ & {$[\mathrm{M}-\mathrm{H}]^{-}$} & 395.0819 & 395.0806 & 3.29 & 9 & Methyoxyastraisoflavan sulfate 2 & $\Delta$ & & $\Delta$ \\
\hline M94 & 20.252 & $\mathrm{C}_{17} \mathrm{H}_{18} \mathrm{O}_{9} \mathrm{~S}$ & {$[\mathrm{M}-\mathrm{H}]^{-}$} & 397.0602 & 397.0599 & 0.76 & 9 & Hydroxyastraisoflavan sulfate 1 & & & $\Delta$ \\
\hline M95 & 49.543 & $\mathrm{C}_{17} \mathrm{H}_{18} \mathrm{O}_{9} \mathrm{~S}$ & {$[\mathrm{M}-\mathrm{H}]^{-}$} & 397.0603 & 397.0599 & 1.01 & 9 & Hydroxyastraisoflavan sulfate 2 & & & $\Delta$ \\
\hline M96 & 44.008 & $\mathrm{C}_{17} \mathrm{H}_{18} \mathrm{O}_{9} \mathrm{~S}$ & {$[\mathrm{M}-\mathrm{H}]^{-}$} & 397.0608 & 397.0599 & 2.27 & 9 & Hydroxyastraisoflavan sulfate 3 & $\Delta$ & $\Delta$ & \\
\hline $\mathrm{M} 97^{\Delta}$ & 51.435 & $\mathrm{C}_{17} \mathrm{H}_{18} \mathrm{O}_{9} \mathrm{~S}$ & {$[\mathrm{M}-\mathrm{H}]^{-}$} & 397.0614 & 397.0599 & 3.78 & 9 & Hydroxyastraisoflavan sulfate 4 & $\Delta$ & & \\
\hline M98 $\triangle$ & 68.372 & $\mathrm{C}_{17} \mathrm{H}_{18} \mathrm{O}_{9} \mathrm{~S}$ & {$[\mathrm{M}-\mathrm{H}]^{-}$} & 397.0620 & 397.0599 & 5.29 & 9 & Hydroxyastraisoflavan sulfate 5 & $\Delta$ & & \\
\hline M99 $\Delta$ & 18.817 & $\mathrm{C}_{17} \mathrm{H}_{18} \mathrm{O}_{9} \mathrm{~S}$ & {$[\mathrm{M}-\mathrm{H}]^{-}$} & 397.0622 & 397.0599 & 5.79 & 9 & Hydroxyastraisoflavan sulfate 6 & & & $\Delta$ \\
\hline M100 $\triangle$ & 62.393 & $\mathrm{C}_{18} \mathrm{H}_{20} \mathrm{O}_{9} \mathrm{~S}$ & {$[\mathrm{M}-\mathrm{H}]^{-}$} & 411.0743 & 411.0755 & -2.92 & 9 & Methoxyastraisoflavan sulfate 1 & $\Delta$ & & \\
\hline M101 $\Delta$ & 60.210 & $\mathrm{C}_{18} \mathrm{H}_{20} \mathrm{O}_{9} \mathrm{~S}$ & {$[\mathrm{M}-\mathrm{H}]^{-}$} & 411.0758 & 411.0755 & 0.73 & 9 & Methoxyastraisoflavan sulfate 2 & $\Delta$ & & \\
\hline M102 $\triangle$ & 58.702 & $\mathrm{C}_{23} \mathrm{H}_{26} \mathrm{O}_{11}$ & {$[\mathrm{M}-\mathrm{H}]^{-}$} & 477.1429 & 477.1402 & 5.66 & 11 & Astraisoflavan-7-O-glucuronide & & $\Delta$ & \\
\hline M103 $\triangle$ & 56.868 & $\mathrm{C}_{23} \mathrm{H}_{26} \mathrm{O}_{11}$ & {$[\mathrm{M}-\mathrm{H}]^{-}$} & 477.1430 & 477.1402 & 5.87 & 11 & Astraisoflavan-2'-O-glucuronide & $\Delta$ & $\Delta$ & \\
\hline M104 $\triangle, \star$ & 38.250 & $\mathrm{C}_{23} \mathrm{H}_{26} \mathrm{O}_{14} \mathrm{~S}$ & {$[\mathrm{M}-\mathrm{H}]^{-}$} & 557.0997 & 557.0970 & 4.85 & 11 & Astraisoflavan glucuronide sulfate 1 & & $\Delta$ & \\
\hline M105 $\Delta, \star$ & 52.510 & $\mathrm{C}_{23} \mathrm{H}_{26} \mathrm{O}_{14} \mathrm{~S}$ & {$[\mathrm{M}-\mathrm{H}]^{-}$} & 557.1001 & 557.0970 & 5.56 & 11 & Astraisoflavan glucuronide sulfate 2 & $\Delta$ & & \\
\hline $\mathrm{M} 106^{\triangle}$ & 35.633 & $\mathrm{C}_{29} \mathrm{H}_{36} \mathrm{O}_{16}$ & {$[\mathrm{M}-\mathrm{H}]^{-}$} & 639.1949 & 639.1931 & 2.82 & 12 & Astraisoflavan-7-O-glucoside-2'-O-glucuronide & $\Delta$ & $\Delta$ & \\
\hline M107 & 41.557 & $\mathrm{C}_{15} \mathrm{H}_{10} \mathrm{O}_{7} \mathrm{~S}$ & {$[\mathrm{M}-\mathrm{H}]^{-}$} & 333.0074 & 333.0074 & 0 & 11 & Daidzein-4'-O-sulfate & $\bar{\Delta}$ & $\bar{\Delta}$ & \\
\hline M108 & 28.925 & $\mathrm{C}_{15} \mathrm{H}_{10} \mathrm{O}_{7} \mathrm{~S}$ & {$[\mathrm{M}-\mathrm{H}]^{-}$} & 333.0080 & 333.0074 & 1.80 & 11 & Daidzein-7-O-sulfate & & $\Delta$ & \\
\hline M109 $\triangle$ & 28.400 & $\mathrm{C}_{15} \mathrm{H}_{10} \mathrm{O}_{10} \mathrm{~S}_{2}$ & {$[\mathrm{M}-\mathrm{H}]^{-}$} & 412.9658 & 412.9643 & 3.63 & 11 & Daidzein-7,4'-O-disulfate & $\Delta$ & $\Delta$ & \\
\hline M110 $\Delta$ & 23.817 & $\mathrm{C}_{21} \mathrm{H}_{18} \mathrm{O}_{10}$ & {$[\mathrm{M}-\mathrm{H}]^{-}$} & 429.0835 & 429.0827 & 1.86 & 13 & Daidzein glucuronide & & $\Delta$ & \\
\hline M111 $\Delta$ & 20.167 & $\mathrm{C}_{21} \mathrm{H}_{18} \mathrm{O}_{13} \mathrm{~S}$ & {$[\mathrm{M}-\mathrm{H}]^{-}$} & 509.0403 & 509.0395 & 1.57 & 13 & Daidzein glucuronide sulfate & & $\Delta$ & \\
\hline M112 $\Delta$ & 44.292 & $\mathrm{C}_{15} \mathrm{H}_{12} \mathrm{O}_{7} \mathrm{~S}$ & {$[\mathrm{M}-\mathrm{H}]^{-}$} & 335.0212 & 335.0231 & -5.67 & 10 & Dihydrodaidzein sulfate & $\Delta$ & & \\
\hline M113 & 53.852 & $\mathrm{C}_{15} \mathrm{H}_{14} \mathrm{O}_{7} \mathrm{~S}$ & {$[\mathrm{M}-\mathrm{H}]^{-}$} & 337.0389 & 337.0387 & 0.59 & 9 & Tetrahydrodaidzein sulfate 1 & $\Delta$ & & \\
\hline M114 & 39.333 & $\mathrm{C}_{15} \mathrm{H}_{14} \mathrm{O}_{7} \mathrm{~S}$ & {$[\mathrm{M}-\mathrm{H}]^{-}$} & 337.0400 & 337.0387 & 3.86 & 9 & Tetrahydrodaidzein sulfate 2 & $\Delta$ & & \\
\hline M115 & 57.002 & $\mathrm{C}_{15} \mathrm{H}_{14} \mathrm{O}_{7} \mathrm{~S}$ & {$[\mathrm{M}-\mathrm{H}]^{-}$} & 337.0402 & 337.0387 & 4.45 & 9 & Tetrahydrodaidzein sulfate 3 & $\Delta$ & $\Delta$ & \\
\hline M116 & 60.560 & $\mathrm{C}_{15} \mathrm{H}_{14} \mathrm{O}_{7} \mathrm{~S}$ & {$[\mathrm{M}-\mathrm{H}]^{-}$} & 337.0409 & 337.0387 & 6.53 & 9 & Tetrahydrodaidzein sulfate 4 & $\Delta$ & & \\
\hline $\mathrm{M} 117^{\Delta}$ & 34.458 & $\mathrm{C}_{15} \mathrm{H}_{14} \mathrm{O}_{10} \mathrm{~S}_{2}$ & {$[\mathrm{M}-\mathrm{H}]^{-}$} & 416.9988 & 416.9956 & 7.67 & 9 & Tetrahydrodaidzein disulfate & $\Delta$ & & \\
\hline M118 & 40.017 & $\mathrm{C}_{15} \mathrm{H}_{10} \mathrm{O}_{5}$ & {$[\mathrm{M}+\mathrm{H}]^{+}$} & 271.0617 & 271.0601 & 5.90 & 11 & Gensitein & $\Delta$ & & \\
\hline M119 & 41.833 & $\mathrm{C}_{15} \mathrm{H}_{10} \mathrm{O}_{8} \mathrm{~S}$ & {$[\mathrm{M}-\mathrm{H}]^{-}$} & 349.0033 & 349.0024 & 2.58 & 11 & Genistein sulfate 1 & $\Delta$ & & \\
\hline M120 & 57.918 & $\mathrm{C}_{15} \mathrm{H}_{10} \mathrm{O}_{8} \mathrm{~S}$ & {$[\mathrm{M}-\mathrm{H}]^{-}$} & 349.0039 & 349.0024 & 4.30 & 11 & Genistein sulfate 2 & $\Delta$ & & \\
\hline M121 & 37.233 & $\mathrm{C}_{15} \mathrm{H}_{10} \mathrm{O}_{8} \mathrm{~S}$ & {$[\mathrm{M}-\mathrm{H}]^{-}$} & 349.0030 & 349.0024 & 1.72 & 11 & Genistein sulfate 3 & $\Delta$ & & \\
\hline M122 $\Delta$ & 25.042 & $\mathrm{C}_{21} \mathrm{H}_{18} \mathrm{O}_{14} \mathrm{~S}$ & {$[\mathrm{M}-\mathrm{H}]^{-}$} & 525.0367 & 525.0370 & -0.57 & 9 & Genistein glucuronide sulfate & & $\Delta$ & \\
\hline M123 & 33.658 & $\mathrm{C}_{15} \mathrm{H}_{12} \mathrm{O}_{8} \mathrm{~S}$ & {$[\mathrm{M}-\mathrm{H}]^{-}$} & 351.0180 & 351.0180 & 0 & 10 & Dihydrogenistein sulfate 1 & $\Delta$ & & \\
\hline M124 $\triangle$ & 74.098 & $\mathrm{C}_{15} \mathrm{H}_{12} \mathrm{O}_{8} \mathrm{~S}$ & {$[\mathrm{M}-\mathrm{H}]^{-}$} & 351.0184 & 351.0180 & 1.14 & 10 & Dihydrogenistein sulfate 2 & & $\Delta$ & \\
\hline
\end{tabular}


Table 2. Cont

\begin{tabular}{|c|c|c|c|c|c|c|c|c|c|c|c|}
\hline NO. & $t_{R}(\min )$ & Formula (M) & Ion & $\begin{array}{l}\text { Meas. } \\
(\mathrm{m} / \mathrm{z})\end{array}$ & $\begin{array}{l}\text { Pred. } \\
(m / z)\end{array}$ & Diff (ppm) & DBE & Identification & Urine & Plasma & Feces \\
\hline M125 $\triangle$ & 51.688 & $\mathrm{C}_{15} \mathrm{H}_{12} \mathrm{O}_{8} \mathrm{~S}$ & {$[\mathrm{M}-\mathrm{H}]^{-}$} & 351.0189 & 351.018 & 2.56 & 10 & Dihydrogenistein sulfate 3 & $\Delta$ & & \\
\hline M126 & 44.758 & $\mathrm{C}_{15} \mathrm{H}_{14} \mathrm{O}_{5}$ & {$[\mathrm{M}-\mathrm{H}]^{-}$} & 273.0757 & 273.0768 & -4.03 & 9 & Tetrahydrogenistein & $\Delta$ & & \\
\hline M127 & 63.985 & $\mathrm{C}_{15} \mathrm{H}_{14} \mathrm{O}_{8} \mathrm{~S}$ & {$[\mathrm{M}-\mathrm{H}]^{-}$} & 353.0359 & 353.0337 & 6.23 & 9 & Tetrahydrogenistein sulfate 1 & $\Delta$ & & $\Delta$ \\
\hline M128 & 65.410 & $\mathrm{C}_{15} \mathrm{H}_{14} \mathrm{O}_{8} \mathrm{~S}$ & {$[\mathrm{M}-\mathrm{H}]^{-}$} & 353.0360 & 353.0337 & 6.51 & 9 & Tetrahydrogenistein sulfate 2 & $\Delta$ & & \\
\hline M129 $\Delta, \star$ & 49.090 & $\mathrm{C}_{20} \mathrm{H}_{22} \mathrm{O}_{12} \mathrm{~S}$ & {$[\mathrm{M}-\mathrm{H}]^{-}$} & 485.0787 & 485.0759 & 5.77 & 10 & Tetrahydrogenistein pentose sulfate & $\Delta$ & & \\
\hline M130 $\Delta, \star$ & 39.850 & $\mathrm{C}_{21} \mathrm{H}_{24} \mathrm{O}_{13} \mathrm{~S}$ & {$[\mathrm{M}-\mathrm{H}]^{-}$} & 515.0911 & 515.0865 & 8.93 & 10 & Tetrahydrogenistin sulfate & $\Delta$ & & \\
\hline M131 & 62.235 & $\mathrm{C}_{15} \mathrm{H}_{14} \mathrm{O}_{6} \mathrm{~S}$ & {$[\mathrm{M}-\mathrm{H}]^{-}$} & 321.0439 & 321.0438 & 0.31 & 9 & Equol sulfate isomer & $\Delta$ & & \\
\hline M132 & 56.152 & $\mathrm{C}_{15} \mathrm{H}_{14} \mathrm{O}_{6} \mathrm{~S}$ & {$[\mathrm{M}-\mathrm{H}]^{-}$} & 321.0444 & 321.0438 & 1.87 & 9 & Equol sulfate 1 & $\Delta$ & $\Delta$ & \\
\hline M133 & 58.943 & $\mathrm{C}_{15} \mathrm{H}_{14} \mathrm{O}_{6} \mathrm{~S}$ & {$[\mathrm{M}-\mathrm{H}]^{-}$} & 321.0454 & 321.0438 & 4.98 & 9 & Equol sulfate 2 & $\Delta$ & & \\
\hline M134 ${ }^{\Delta}$ & 46.017 & $\mathrm{C}_{21} \mathrm{H}_{22} \mathrm{O}_{9}$ & {$[\mathrm{M}-\mathrm{H}]^{-}$} & 417.1185 & 417.1191 & -1.44 & 11 & Equol glucuronide 1 & $\Delta$ & $\Delta$ & \\
\hline M135 $\triangle$ & 47.975 & $\mathrm{C}_{21} \mathrm{H}_{22} \mathrm{O}_{9}$ & {$[\mathrm{M}-\mathrm{H}]^{-}$} & 417.1209 & 417.1191 & 4.32 & 11 & Equol glucuronide 2 & $\Delta$ & $\Delta$ & \\
\hline M136 $\triangle, \star$ & 32.293 & $\mathrm{C}_{21} \mathrm{H}_{22} \mathrm{O}_{12} \mathrm{~S}$ & {$[\mathrm{M}-\mathrm{H}]^{-}$} & 497.0786 & 497.0759 & 5.43 & 11 & Equol glucuronide sulfate 1 & & $\Delta$ & \\
\hline M137 $\Delta, \star$ & 28.058 & $\mathrm{C}_{21} \mathrm{H}_{22} \mathrm{O}_{12} \mathrm{~S}$ & {$[\mathrm{M}-\mathrm{H}]^{-}$} & 497.0797 & 497.0759 & 7.64 & 11 & Equol glucuronide sulfate 2 & & $\Delta$ & \\
\hline $\mathrm{M} 138^{\Delta}$ & 37.317 & $\mathrm{C}_{21} \mathrm{H}_{22} \mathrm{O}_{12} \mathrm{~S}$ & {$[\mathrm{M}-\mathrm{H}]^{-}$} & 497.0757 & 497.0759 & -0.4 & 11 & Equol glucuronide sulfate 3 & & $\Delta$ & \\
\hline M139 $\triangle$ & 57.977 & $\mathrm{C}_{15} \mathrm{H}_{16} \mathrm{O}_{6} \mathrm{~S}$ & {$[\mathrm{M}-\mathrm{H}]^{-}$} & 323.0616 & 323.0595 & 6.5 & 8 & Dihydroequol sulfate & $\Delta$ & & \\
\hline M140 & 28.817 & $\mathrm{C}_{16} \mathrm{H}_{14} \mathrm{O}_{6}$ & {$[\mathrm{M}+\mathrm{H}]^{+}$} & 303.0878 & 303.0863 & 4.95 & 10 & Dihydropratensein & $\Delta$ & & \\
\hline M141 $\triangle$ & 24.925 & $\mathrm{C}_{22} \mathrm{H}_{20} \mathrm{O}_{15} \mathrm{~S}$ & {$[\mathrm{M}-\mathrm{H}]^{-}$} & 555.0487 & 555.0450 & 6.67 & 13 & Pratensein glucuronide sulfate & & $\Delta$ & \\
\hline M142 $\Delta, \star$ & 26.300 & $\mathrm{C}_{27} \mathrm{H}_{30} \mathrm{O}_{17}$ & {$[\mathrm{M}-\mathrm{H}]^{-}$} & 625.1462 & 625.1410 & 8.32 & 13 & Tetrahydro trihydroxyisoflavone diglucuronide 1 & $\Delta$ & & \\
\hline M143 $\triangle, \star$ & 26.867 & $\mathrm{C}_{27} \mathrm{H}_{30} \mathrm{O}_{17}$ & {$[\mathrm{M}-\mathrm{H}]^{-}$} & 625.1467 & 625.1410 & 9.12 & 13 & Tetrahydro trihydroxyisoflavone diglucuronide 2 & $\Delta$ & & \\
\hline M144 $\triangle$ & 41.650 & $\mathrm{C}_{15} \mathrm{H}_{14} \mathrm{O}_{9} \mathrm{~S}$ & {$[\mathrm{M}-\mathrm{H}]^{-}$} & 369.0303 & 369.0286 & 4.61 & 9 & Tetrahydro-tetrahydroxyisoflavone sulfate & $\Delta$ & & \\
\hline M145 ${ }^{\Delta}$ & 41.930 & $\mathrm{C}_{22} \mathrm{H}_{20} \mathrm{O}_{15} \mathrm{~S}$ & {$[\mathrm{M}-\mathrm{H}]^{-}$} & 555.0478 & 555.0450 & 6.76 & 13 & Pratensein glucuronide sulfate & & & \\
\hline & 61.012 & $\mathrm{C}_{22} \mathrm{H}_{20} \mathrm{O}_{11}$ & {$[\mathrm{M}-\mathrm{H}]^{-}$} & 459.0925 & 459.0933 & -1.23 & 13 & Calycosin-7-O-glucuronide & & & \\
\hline \multirow[t]{2}{*}{ M147 $\triangle$} & 69.988 & $\mathrm{C}_{17} \mathrm{H}_{18} \mathrm{O}_{8} \mathrm{~S}$ & {$[\mathrm{M}-\mathrm{H}]^{-}$} & 381.0652 & 381.0650 & 0.52 & 9 & Astraisoflavan sulfate isomer & & & \\
\hline & & & & & Sum & & & & 106 & 64 & 17 \\
\hline
\end{tabular}

Note: $t_{R}$ : Retention time; Meas.: measured; Pred.: predicted; Diff: difference; DBE: double bone equivalents. ${ }^{\triangle}$ New metabolites found in vivo after administration of ARTF; ${ }^{\star}$ Potential New compound by retrieving information from SciFinder database. $\mathbf{\Lambda}$ Detected. 
Identification of the Sulfates of the Ring Cleavage Products of Flavone (M1-M16)

A total of 16 compounds were assigned as metabolites originating from flavone, which underwent ring cleavage, then conjugation with sulfuric acid, and all of them were new metabolites of ARTF. M1 showed $[\mathrm{M}-\mathrm{H}]^{-}$at $\mathrm{m} / \mathrm{z} 200.99$ and its molecular formula was predicted as $\mathrm{C}_{7} \mathrm{H}_{6} \mathrm{O}_{5} \mathrm{~S}$, and the fragment ion at $m / z 121.03$ was formed by the neutral loss of $79.95 \mathrm{Da}\left(\mathrm{SO}_{3}\right)$ in its $\mathrm{MS}^{2}$ spectra, so it was determined as hydroxyl-benzaladehyde sulfate or other isomers. M2 showed $[\mathrm{M}-\mathrm{H}]^{-}$at $m / z$ 219.00 and its molecular formula was predicted as $\mathrm{C}_{7} \mathrm{H}_{8} \mathrm{O}_{6} \mathrm{~S}$, and the fragment ion generated by loss $79.95 \mathrm{Da}$ could be detected at $\mathrm{m} / \mathrm{z}$ 139.04, which was predicted as $\mathrm{C}_{7} \mathrm{H}_{8} \mathrm{O}_{3}$ and was one $\mathrm{CH}_{2}$ more than pyrogallol, so it was tentatively identified as methyl pyrogallol sulfate [24] or other isomers. M3 and M4 showed $[\mathrm{M}-\mathrm{H}]^{-}$at $m / z 231.00$, which indicated that their molecular formulae were $\mathrm{C}_{8} \mathrm{H}_{8} \mathrm{O}_{6} \mathrm{~S}$. In the MS ${ }^{2}$ spectra, fragment ions at $m / z$ 151.04, 137.03 were formed by sequential losses of $\mathrm{SO}_{3}$ and $\mathrm{CH}_{2}$. According to literature, they were identified as hydroxy phenylacetic acid sulfate [25]. M5 and M6 showed $[\mathrm{M}-\mathrm{H}]^{-}$at $m / z 243.00$, which indicated that their molecular formulae were $\mathrm{C}_{9} \mathrm{H}_{8} \mathrm{O}_{6} \mathrm{~S}$, and according to the fragment ions at $m / z 163.04,119.05$ and previous report, they were identified as hydroxycinnamic acid sulfate [26]. M7 showed [M- H] $]^{-}$at $m / z 243.03$ and was predicted as $\mathrm{C}_{10} \mathrm{H}_{12} \mathrm{O}_{5} \mathrm{~S}$. And according to the fragment ions at $m / z$ 163.08, 148.05 in $\mathrm{MS}^{2}$ spectra, it was regarded as eugenol sulfate [27]. M8 showed [M - H] $]^{-}$at $m / z 247.00$ and was predicted as $\mathrm{C}_{8} \mathrm{H}_{8} \mathrm{O}_{7} \mathrm{~S}$, and yielded a fragment ion at $m / z 167.03$ by neutral loss of $79.95 \mathrm{Da}\left(\mathrm{SO}_{3}\right)$, so it was determined as vanillic acid sulfate [28]. M9 showed $[\mathrm{M}-\mathrm{H}]^{-}$at $m / z 247.03$, which indicated that their molecular formulae were $\mathrm{C}_{9} \mathrm{H}_{12} \mathrm{O}_{6} \mathrm{~S}$, and according to the fragment ions at $m / z$ 167.07, 153.09, 137.05 and previous report, it was identified as homovanillyl alcohol sulfate [29]. M10 showed [M- H] $]^{-}$at $m / z$ 273.00, which indicated that its molecular formula was $\mathrm{C}_{10} \mathrm{H}_{10} \mathrm{O}_{7} \mathrm{~S}$, and according to the fragment ions at $m / z 178.03,134.04$, it was identified as ferulic acid sulfate [24]. M11 and M12 showed [M- H] $]^{-}$at $m / z$ 273.04, which indicated that their molecular formulae were $\mathrm{C}_{11} \mathrm{H}_{14} \mathrm{O}_{6} \mathrm{~S}$. And the fragment ions at $\mathrm{m} / z \mathrm{z} 193.09,178.06,163.03$ were observed in $\mathrm{MS}^{2}$ spectra, which were $30.01 \mathrm{Da}\left(\mathrm{OCH}_{2}\right)$ lager than that of the aglycon of M7. Therefore, they were tentatively determined as methoxyeugenol sulfate. M16 showed $[\mathrm{M}-\mathrm{H}]^{-}$at $m / z$ 303.02, which indicated that its molecular formula was $\mathrm{C}_{11} \mathrm{H}_{12} \mathrm{O}_{8} \mathrm{~S}$, and the fragment ions at $m / z$ 223.06, $208.04,164.05,149.02$, were similar to the fragment ions of 4-hydroxy-3,5-dimethoxycinnamic acid, so it was determined as 4-hydroxy-3,5-dimethoxycinnamic acid sulfate [30].

\section{Identification of the Calycosin-Related Metabolites (M17-M72, M146)}

M17 showed [M - H] $]^{-}$at $m / z 283.06$ and its molecular formula was predicted as $\mathrm{C}_{16} \mathrm{H}_{12} \mathrm{O}_{5}$. The fragment ions at $m / z$ 269.04, 268.04, 195.04 were detected in $\mathrm{MS}^{2}$, which were like those of calycosin, so it was identified as calycosin isomer. M18-M20 were identified as calycosin isomer owing that their predicted molecular formulae and fragment ions were like those of calycosin in the positive ion mode. M21 showed $[\mathrm{M}+\mathrm{H}]^{+}$at 301.06 and its molecule formula was predicted as $\mathrm{C}_{16} \mathrm{H}_{12} \mathrm{O}_{6}$, which had one more oxygen atom than that of calycosin, and the fragment ions at $m / z 270.08,197.08$ were similar to those of calycosin in positive ion mode, so it was determined as hydroxycalycosin. The fragment ion of [aglycon-H] ${ }^{-}$at $m / z 283.06$ could be detected in the $\mathrm{MS}^{2}$ spectra of M25-M34, which was predicted as $\mathrm{C}_{16} \mathrm{H}_{12} \mathrm{O}_{5}$, and other fragment ions were like those of calycosin, so they were determined as calycosin metabolites. M25-M30 were predicted as $\mathrm{C}_{16} \mathrm{H}_{12} \mathrm{O}_{8} \mathrm{~S}$ according to $[\mathrm{M}-\mathrm{H}]^{-}$ at $m / z 363.02$, and the [aglycon $-\mathrm{H}]^{-}$formed by the neutral loss of $79.95 \mathrm{Da}\left(\mathrm{SO}_{3}\right)$ was detected. Therefore, they were determined as calycosin sulfate and its isomers. In the same way, M30 was determined as calycosin-7,3'-O-disulfate. M31 and M146 showed [M - H] $]^{-}$at $m / z 459.09$ and their molecular formulae were predicted as $\mathrm{C}_{22} \mathrm{H}_{20} \mathrm{O}_{11}$, and the fragment ion of [aglycon - $\left.\mathrm{H}\right]^{-}$were formed by the neutral loss of $176.03 \mathrm{Da}\left(\mathrm{C}_{6} \mathrm{H}_{8} \mathrm{O}_{6}\right)$ in the $\mathrm{MS}^{2}$ spectra, so they were determined as calycosin glucuronide. There are two positions $\left(\mathrm{C}-7-\mathrm{OH}\right.$ and $\left.\mathrm{C}-3^{\prime}-\mathrm{OH}\right)$ in calycosin that could be linked with glucuronic acid, and C-3'-OH was the major position according to a previous study [31]. In addition, M31 was the main metabolite, hence it was determined as calycosin-3'-O-glucuronide, and M146 was determined as calycosin-7-O-glucuronide. 
In the MS ${ }^{2}$ spectra of $\mathbf{M 3 5}-\mathbf{M} 39$, the fragment ion of [aglycon - H] ${ }^{-}$at $m / z 299.05$ can be detected, which were predicted as $\mathrm{C}_{16} \mathrm{H}_{11} \mathrm{O}_{6}$ and was the same as M21, so they were tentatively determined as metabolites of hydroxycalycosin. And according to molecule formulae and characteristic neutral losses, they were tentatively determined as hydroxycalycosin sulfate or glucuronide, respectively.

M42 showed $[\mathrm{M}-\mathrm{H}]^{-}$at $\mathrm{m} / \mathrm{z} 621.15$ and its molecular formula was predicted as $\mathrm{C}_{28} \mathrm{H}_{30} \mathrm{O}_{16}$. The fragment ion at $m / z 283.06$ predicted as $\mathrm{C}_{16} \mathrm{H}_{11} \mathrm{O}_{5}$ was detected in $\mathrm{MS}^{2}$ which was generated by sequential loss of $162.05 \mathrm{Da}\left(\mathrm{C}_{6} \mathrm{H}_{10} \mathrm{O}_{5}\right)$, and $176.03 \mathrm{Da}\left(\mathrm{C}_{6} \mathrm{H}_{8} \mathrm{O}_{6}\right)$. Since calycosin-7-O-glucoside was the main constituent of ARTF, so it was determined as calycosin-7-O-glucoside-3'-O-glucuronide.

The fragment ion of [aglycon $-\mathrm{H}^{-}$at $m / z 285.08$ could be detected in the $\mathrm{MS}^{2}$ spectra of M43-M46, which was predicted as $\mathrm{C}_{16} \mathrm{H}_{13} \mathrm{O}_{5}$ and had $2 \mathrm{H}(2.01 \mathrm{Da})$ more than that of calycosin, so they were tentatively regarded as metabolites of dihydrocalycosin. The fragment ion of [aglycon $-\mathrm{H}]^{-}$at $m / z$ 287.09 could be detected in the $\mathrm{MS}^{2}$ spectra of $\mathbf{M} 54-\mathbf{M} 63$, which were predicted as $\mathrm{C}_{16} \mathrm{H}_{15} \mathrm{O}_{5}$ and had $4 \mathrm{H}$ more than that of calycosin, so they were tentatively regarded as metabolites of tetrahydrocalycosin. In the $\mathrm{MS}^{2}$ spectra of $\mathbf{M 6 4 - M 6 9 , ~ t h e ~ f r a g m e n t ~ i o n ~ o f ~ [ a g l y c o n ~ - ~ H ] ~}{ }^{-}$at $m / z 303.08$ could be detected, which were predicted as $\mathrm{C}_{16} \mathrm{H}_{15} \mathrm{O}_{6}$ and had one more oxygen atom than that of tetrahydrocalycosin, so they were tentatively regarded as hydroxy tetrahydrocalycosin metabolites.

\section{Identification of the Formononetin-Related Metabolites (M73-M78)}

M73 showed $[\mathrm{M}-\mathrm{H}]^{-}$at $m / z 347.02$ and its molecular formula was predicted as $\mathrm{C}_{16} \mathrm{H}_{12} \mathrm{O}_{7} \mathrm{~S}$. The fragment ion of $\mathrm{m} / \mathrm{z} 267.07$ in the $\mathrm{MS}^{2}$ spectra was formed by the neutral loss of $79.95\left(\mathrm{SO}_{3}\right)$, which was predicted as $\mathrm{C}_{16} \mathrm{H}_{11} \mathrm{O}_{4}$, and its fragment ion $m / z 252.03$ was like that of formononetin. Because only C-7-OH could be sulfated, so $\mathbf{M 7 3}$ was determined as formononetin-7-O-sulfate. In the same way, M74 was determined as formononetin-7-O-glucuronide. M75-M77 showed [M - H] ${ }^{-}$at $m / z 351.05$ and their molecular formulae were predicted as $\mathrm{C}_{16} \mathrm{H}_{16} \mathrm{O}_{7} \mathrm{~S}$. The fragment ions at $m / z 271.09$ formed by neutral loss of $79.95 \mathrm{Da}\left(\mathrm{SO}_{3}\right)$ was determined as $\mathrm{C}_{16} \mathrm{H}_{15} \mathrm{O}_{4}$, which had $4 \mathrm{H}$ more than that of formononetin, so they were tentatively determined as tetrahydroformononetin sulfate.

\section{Identification of the Astrapterocarpan-Related Metabolites (M79-M84)}

M79 showed $[\mathrm{M}-\mathrm{H}]^{-}$at $m / z 379.05$ and its molecular formula was predicted as $\mathrm{C}_{17} \mathrm{H}_{16} \mathrm{O}_{8} \mathrm{~S}$. The fragment ions $m / z 299.08$ formed by a natural loss of $176.03 \mathrm{Da}\left(\mathrm{C}_{6} \mathrm{H}_{8} \mathrm{O}_{6}\right)$ was predicted as $\mathrm{C}_{17} \mathrm{H}_{15} \mathrm{O}_{5}$, which was the same to that of astrapterocarpan. Owing that only $\mathrm{C}-3-\mathrm{OH}$ of astrapterocarpan could be linked to sulfuric acid, it was determined as astrapterocarpan-3-O-sulfate. In the same way, M80 was determined as astrapterocarpan-3-O-glucuronide.

M82-M84 showed $[\mathrm{M}-\mathrm{H}]^{-}$at $m / z 491.12$ and their molecular formulae were predicted as $\mathrm{C}_{23} \mathrm{H}_{24} \mathrm{O}_{12}$. The fragment ions at $m / z 315.09$ generated by neutral loss of $176.03 \mathrm{Da}\left(\mathrm{C}_{6} \mathrm{H}_{8} \mathrm{O}_{6}\right)$ was predicted as $\mathrm{C}_{17} \mathrm{H}_{15} \mathrm{O}_{6}$, and had one more $\mathrm{O}$ than that of astrapterocarpan, so they were tentatively determined as hydroxyastrapterocarpan glucuronide.

\section{Identification of the Astraisoflavan-Related Metabolites (M85-M106, M147)}

M85 showed $[\mathrm{M}+\mathrm{H}]^{+}$at $m / z 303.13$ and its molecular formula was predicted as $\mathrm{C}_{17} \mathrm{H}_{18} \mathrm{O}_{5}$. The fragment ions at $m / z$ 167.10, 149.09, and 125.07 could be detected in $\mathrm{MS}^{2}$ spectra and were like those of astraisoflavan in positive ion mode. So, M85 was determined as astraisoflavan isomer.

M102-M103 showed [M - H] $]^{-}$at $m / z 477.14$ and their molecular formulae were predicted as $\mathrm{C}_{23} \mathrm{H}_{26} \mathrm{O}_{11}$. The fragment ions at $\mathrm{m} / \mathrm{z} 301.10$ in $\mathrm{MS}^{2}$ spectra formed by a neutral loss of $176.03 \mathrm{Da}$ $\left(\mathrm{C}_{6} \mathrm{H}_{8} \mathrm{O}_{6}\right)$ was the same to that of astraisoflavan. Therefore, they were determined as astraisoflavan glucuronide. Because there are only two glucuronidation sites (C-7-OH, C-2'-OH) in astraisoflavan, and a larger CLogP value means a lower polarity and a larger retention time in reversed phase HPLC, M102 $\left(C \log \mathrm{P}=3.6083, \mathrm{t}_{\mathrm{R}}=58.702 \mathrm{~min}\right)$ was determined as astraisoflavan-7-O-glucuronide and M103 $\left(\mathrm{CLog} P=3.2673, \mathrm{t}_{\mathrm{R}}=57.802 \mathrm{~min}\right)$ was determined as astraisoflavan-2' $-\mathrm{O}$-glucuronide. 
Identification of the Daidzein-Related Metabolites (M107-M117)

M107 and M108 showed $[\mathrm{M}-\mathrm{H}]^{-}$at $m / z 333.00$ and their molecular formulae were predicted as $\mathrm{C}_{15} \mathrm{H}_{10} \mathrm{O}_{7} \mathrm{~S}$. The fragment ion at $\mathrm{m} / z 253.05$ in $\mathrm{MS}^{2}$ spectra formed by the neutral loss of $79.95 \mathrm{Da}\left(\mathrm{SO}_{3}\right)$, and it was predicted as daidzein owing that the fragment was predicted as $\mathrm{C}_{15} \mathrm{H}_{9} \mathrm{O}_{4}$ and $m / z 225.05$, $197.06,135.01$ were detected in $\mathrm{MS}^{3}$ spectra. Because there are two sulfation sites $\left(\mathrm{C}-7-\mathrm{OH}, \mathrm{C}-4^{\prime}-\mathrm{OH}\right)$ in daidzein, and a larger CLogP value means a lower polarity and a larger retention time in reversed phase HPLC, M107 (CLogP $\left.=0.4985, \mathrm{t}_{\mathrm{R}}=41.557 \mathrm{~min}\right)$ was determined as daidzein-4'-O-sulfate and M108 (CLogP $\left.=0.3050, t_{R}=28.925 \mathrm{~min}\right)$ was determined as daidzein-7-O-sulfate.

M112 showed $[\mathrm{M}-\mathrm{H}]^{-}$at $m / z 335.02$ and its molecular formula was predicted as $\mathrm{C}_{15} \mathrm{H}_{12} \mathrm{O}_{7} \mathrm{~S}$. In $\mathrm{MS}^{2}$ spectra, the fragment ion at $m / z 255.06$ was formed by the neutral loss of $79.95 \mathrm{Da}\left(\mathrm{SO}_{3}\right)$, which was predicted as $\mathrm{C}_{15} \mathrm{H}_{11} \mathrm{O}_{4}$ and had $2 \mathrm{H}$ more than that of daidzein. Therefore, it was tentatively determined as dihydrodaidzein sulfate.

M113-M116 showed $[\mathrm{M}-\mathrm{H}]^{-}$at $m / z 337.04$ and their molecular formulae were predicted as $\mathrm{C}_{15} \mathrm{H}_{14} \mathrm{O}_{7} \mathrm{~S}$. The fragment ion at $\mathrm{m} / z 257.08$ in $\mathrm{MS}^{2}$ spectra formed by the neutral loss of $79.95 \mathrm{Da}$ $\left(\mathrm{SO}_{3}\right)$, which was predicted as $\mathrm{C}_{15} \mathrm{H}_{13} \mathrm{O}_{4}$ and had $4 \mathrm{H}$ more than that of daidzein. Therefore, they were tentatively determined as tetrahydrodaidzein sulfate.

Identification of the Genistein-Related Metabolites (M118-M130)

M118 showed $[\mathrm{M}+\mathrm{H}]^{+}$at $m / z 271.06$ and its molecule formula was predicted as $\mathrm{C}_{15} \mathrm{H}_{10} \mathrm{O}_{5}$. The fragment ions at $\mathrm{m} / \mathrm{z} 253.01,225.06,215.07$ which were like those of genistein in reported literature [32], so it was determined as genistein.

M123-M125 showed $[\mathrm{M}-\mathrm{H}]^{-}$at $\mathrm{m} / \mathrm{z} 351.02$ and their molecular formulae were predicted as $\mathrm{C}_{15} \mathrm{H}_{12} \mathrm{O}_{8} \mathrm{~S}$. The fragment ion at $m / z 271.06$ was formed by a neutral loss $79.95 \mathrm{Da}\left(\mathrm{SO}_{3}\right)$, which was predicted as $\mathrm{C}_{15} \mathrm{H}_{11} \mathrm{O}_{5}$ and had $2 \mathrm{H}$ more than that of genistein, so they were tentatively determined as dihydrogenistein sulfate.

M126 showed $[\mathrm{M}-\mathrm{H}]^{-}$at $\mathrm{m} / \mathrm{z} 273.07$ and its molecular formula was predicted as $\mathrm{C}_{15} \mathrm{H}_{14} \mathrm{O}_{5}$, which was $4 \mathrm{H}$ more than that of genistein, so it was tentatively determined as tetrahydrogenistein. M130 showed $[\mathrm{M}-\mathrm{H}]^{-}$at $\mathrm{m} / z 515.09$ and its molecules formula was predicted as $\mathrm{C}_{21} \mathrm{H}_{24} \mathrm{O}_{13} \mathrm{~S}$. The fragment ion at $m / z 273.07$ which was predicted as $\mathrm{C}_{15} \mathrm{H}_{13} \mathrm{O}_{5}$ formed by a neutral loss of $162.05 \mathrm{Da}$ $\left(\mathrm{C}_{6} \mathrm{H}_{10} \mathrm{O}_{5}\right)$ and $79.95 \mathrm{Da}\left(\mathrm{SO}_{3}\right)$, so it was tentatively determined as tetrahydrogenistin sulfate.

\section{Identification of the Equol-Related Metabolites (M131-M139)}

In the MS2 spectra of M131-M138, the fragment ion of [aglycon - $\mathrm{H}^{-}$at $m / z 241.09$ could be detected, which were predicted as $\mathrm{C}_{15} \mathrm{H}_{13} \mathrm{O}_{3}$ and its fragment ions of $m / z$ 135.05, 121.04, 119.06 were like those of equol [33], so they were regarded as equol metabolites. M136-M138 showed [M - H] ${ }^{-}$ at $m / z 497.07$ and their molecules formulae were predicted as $\mathrm{C}_{21} \mathrm{H}_{22} \mathrm{O}_{12} \mathrm{~S}$, and the [aglycon $\left.-\mathrm{H}\right]^{-}$ formed by the neutral loss of $176.03 \mathrm{Da}\left(\mathrm{C}_{6} \mathrm{H}_{8} \mathrm{O}_{6}\right), 79.97 \mathrm{Da}\left(\mathrm{SO}_{3}\right)$. Therefore, they were determined as equol glucuronide sulfate. M139 showed $[\mathrm{M}-\mathrm{H}]^{-}$at $m / z 323.06$ and its molecular formula was predicted as $\mathrm{C}_{15} \mathrm{H}_{16} \mathrm{O}_{6} \mathrm{~S}$. The fragment ion at $\mathrm{m} / z 243.10$ formed by a neutral loss of $79.95 \mathrm{Da}\left(\mathrm{SO}_{3}\right)$, which was predicted as $\mathrm{C}_{15} \mathrm{H}_{15} \mathrm{O}_{3}$ and $2 \mathrm{H}(2.01 \mathrm{Da})$ more than that of equol. Hence, it was tentatively determined as dihydroequol sulfate.

Identification of the other Metabolites (M140-M145)

M140 showed $[\mathrm{M}+\mathrm{H}]^{+}$at $\mathrm{m} / \mathrm{z} 303.09$ and its molecular formula was predicted as $\mathrm{C}_{16} \mathrm{H}_{14} \mathrm{O}_{6}$, which had $2 \mathrm{H}$ more than that of pratensein. Therefore, it was tentatively determined as dihydropratensein. M141 and M145 showed $[\mathrm{M}-\mathrm{H}]^{-}$at $\mathrm{m} / z 555.05$ and their molecular formulae were predicted as $\mathrm{C}_{22} \mathrm{H}_{20} \mathrm{O}_{15} \mathrm{~S}$. The fragment ions at $m / z 299.05$ predicted as $\mathrm{C}_{16} \mathrm{H}_{11} \mathrm{O}_{6}$ was formed by a neutral loss of $176.03 \mathrm{Da}\left(\mathrm{C}_{6} \mathrm{H}_{8} \mathrm{O}_{6}\right)$ and $79.95 \mathrm{Da}\left(\mathrm{SO}_{3}\right)$. Therefore, it was tentatively determined as pratensein glucuronide sulfate. 


\subsection{Distribution of Original Constituents and Metabolites of ARTF in Rats Organs}

\subsubsection{Distribution of Original Constituents}

Nineteen original constituents were detected in the organs, with zero in brain, three in heart, five in liver, four in spleen, three in lung, five in kidney, seventeen in stomach, seven in small intestine, seven in colon intestine, and six in thymus, respectively (Table S2, Figure S17a-S25a). Six (F18-F23) were only detected in the organs and were not detected in the urine, plasma, and feces. Calycosin (F1), formononetin (F6), daidzein (F11), and naringin (F18) were widely distributed, which could be detected in seven and even more organs, and these compounds may be important material basis for the efficacy of ARTF.

\subsubsection{Distribution of Metabolites}

Sixty-five metabolites were identified in the organs, of which three metabolites (M145-M147) were only detected in the organs, and $0,3,18,5,6,22,30,46,21$, and 6 were identified in the brain, heart, liver, spleen, lung, kidney, stomach, small intestine, colon, and thymus (Table S3, Figures S17b-S25b), respectively. Twelve metabolites containing seven sulfates and five glucuronides were distributed widely in five and even more tissues. Seven sulfates were calycosin sulfate (M26), tetrahydrocalycosin sulfate (M57, M58), hydroxyastraisoflavan sulfate (M96), daidzein-4'-O-sulfate (M107), equol sulfate (M132, M133), respectively. Five glucuronides consisted of calycosin-3'-O-glucuronide (M32), dimethoxy hydroxytetrahydrocalycosin glucuronide (M72), astrapterocarpan-3-O-glucuronide (M80), astraisoflavan-2'-O-glucuronide (M103), and astraisoflavan-7-O-glucoside-2'-O-glucuronide (M106). These widely distributed metabolites may play an important role in the efficacies of ARTF.

\subsection{Identification of Metabolites Isolated from Rat Urine}

MI-1 (M108) was obtained as a white powder and assigned a molecular formula of $\mathrm{C}_{15} \mathrm{H}_{10} \mathrm{O}_{7} \mathrm{~S}$ based on its HR-ESI-MS mass spectrum, which showed a quasi-molecular ion peak [M - H] $]^{-}$at $m / z$ 333.0076 (calcd. for $\mathrm{C}_{15} \mathrm{H}_{10} \mathrm{O}_{7} \mathrm{~S} 333.0069$ ). The main fragment ion was $\mathrm{m} / z 253.0505\left[\mathrm{M}-\mathrm{SO}_{3}-\mathrm{H}^{-}\right.$ in MS $^{2}$ spectra, so it was regarded as a sulfate. MI-1 (M108): ${ }^{13} \mathrm{C}-\mathrm{NMR}$ (DMSO-d $\left.6,100 \mathrm{MHz}\right) \mathrm{ppm}$ : 153.6 (C-2), 122.5 (C-3), 175.0 (C-4), 129.7 (C-5), 118.0 (C-6), 158.1 (C-7), 107.1 (C-8), 156.6 (C-9), 119.0 (C-10), $123.7\left(\mathrm{C}-1^{\prime}\right), 130.2$ (C-2', C-6'), 115.08 (C-3', C-5'), 157.3 (C-4'), which were in consistent with daidzein [34]. ${ }^{1} \mathrm{H}-\mathrm{NMR}$ spectra of MI-1 (M108) $\left(\mathrm{DMSO}_{6}, 400 \mathrm{MHz}\right)$ ppm: $8.38(1 \mathrm{H}, \mathrm{s}, \mathrm{H}-2), 8.03(1 \mathrm{H}, \mathrm{d}$, $J=8.8 \mathrm{~Hz}, \mathrm{H}-5), 7.43(1 \mathrm{H}, \mathrm{d}, J=2.1 \mathrm{~Hz}, \mathrm{H}-8), 7.40\left(2 \mathrm{H}, \mathrm{d}, J=8.3 \mathrm{~Hz}, \mathrm{H}-2^{\prime}\right.$ and $\left.\mathrm{H}-66^{\prime}\right), 7.25(1 \mathrm{H}, \mathrm{dd}, J=8.8 \mathrm{~Hz}$, $2.1 \mathrm{~Hz}, \mathrm{H}-6), 6.81\left(2 \mathrm{H}, \mathrm{d}, J=8.3 \mathrm{~Hz}, \mathrm{H}-3^{\prime}\right.$ and H-5'), which were like those of daidzein-7-O-sulfate reported in literature [35].Given all of this, MI-1 was determined as daidzein-7-O-sulfate. Its structure and NMR spectroscopy were shown in Figure 3a and Figure S26, respectively.

MI-2 (M32) was obtained as a white powder and assigned a molecular formula of $\mathrm{C}_{22} \mathrm{H}_{20} \mathrm{O}_{11}$ based on its HR-ESI-MS mass spectrum, which showed a quasi-molecular ion peak $[\mathrm{M}-\mathrm{H}]^{-} \mathrm{t} m / z$ 459.0943 (calcd. for $\mathrm{C}_{22} \mathrm{H}_{20} \mathrm{O}_{11} 459.0935$ ). The main fragment ion was $m / z 283.0609\left[\mathrm{M}-\mathrm{C}_{6} \mathrm{H}_{8} \mathrm{O}_{6}-\mathrm{H}\right]^{-}$ in $\mathrm{MS}^{2}$ spectra, so it was regarded as a glucuronide. MI-2 (M32): ${ }^{1} \mathrm{H}-\mathrm{NMR}$ spectra (DMSO-d $\left.\mathrm{d}_{6}, 400 \mathrm{MHz}\right)$ ppm: $8.31(1 \mathrm{H}, \mathrm{s}, \mathrm{H}-2), 7.97(1 \mathrm{H}, \mathrm{d}, J=8.8 \mathrm{~Hz}, \mathrm{H}-5), 6.94(1 \mathrm{H}, \mathrm{dd}, J=8.8 \mathrm{~Hz}, 2.2 \mathrm{~Hz}, \mathrm{H}-6), 6.87(1 \mathrm{H}$, $\mathrm{d}, J=2.2 \mathrm{~Hz}, \mathrm{H}-8), 7.29\left(1 \mathrm{H}, \mathrm{d}, J=2.0 \mathrm{~Hz}, \mathrm{H}-2^{\prime}\right), 7.04\left(1 \mathrm{H}, \mathrm{d}, J=8.5 \mathrm{~Hz}, \mathrm{H}-5^{\prime}\right), 7.23(1 \mathrm{H}, \mathrm{dd}, J=8.5 \mathrm{~Hz}$, $\left.2.0 \mathrm{~Hz}, \mathrm{H}-66^{\prime}\right), 3.79\left(3 \mathrm{H}, \mathrm{s}, \mathrm{C}-4^{\prime}-\mathrm{OCH}_{3}\right), 10.88(1 \mathrm{H}, \mathrm{s}, \mathrm{C}-7-\mathrm{OH}) .{ }^{13} \mathrm{C}-\mathrm{NMR}$ (DMSO-d 6 , 100MHz) ppm: 153.5 (C-2), 123.3 (C-3), 174.7 (C-4), 127.4 (C-5), 115.4 (C-6), 162.7 (C-7), 102.3 (C-8), 157.5 (C-9), 116.4 (C-10), 124.5 (C-1'), 116.7 (C-2'), 145.7 (C-3'), 149.2 (C-4'), $112.6\left(\mathrm{C}-5^{\prime}\right), 123.3$ (C-6'), $55.9\left(\mathrm{C}-4^{\prime}-\mathrm{OCH}_{3}\right)$, which were similar to ${ }^{13} \mathrm{C}-\mathrm{NMR}$ of calycosin reported in literature [36]. The characteristic signals of six carbons in glucuronide were 100.2 (C-1"), 73.1 (C-2"), $76.3\left(\mathrm{C}-3^{\prime \prime}\right), 71.6\left(\mathrm{C}-4^{\prime \prime}\right), 75.6\left(\mathrm{C}-5^{\prime \prime}\right), 170.2\left(\mathrm{C}-6^{\prime \prime}\right)$, and all were consisted with calycosin-3'-O-glucuronide [31]. Based on the above analysis, MI-2 (M32) was determined as calycosin-3'-O-glucuronide. Its structure and NMR spectroscopy were shown in Figure $3 \mathrm{~b}$ and Figure S27, respectively. 


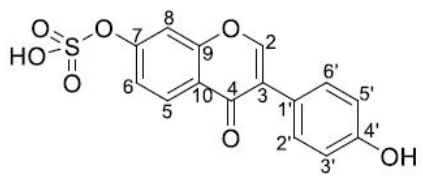

MI-1 (M108): Daidzein-7-O-sulfate

(a)

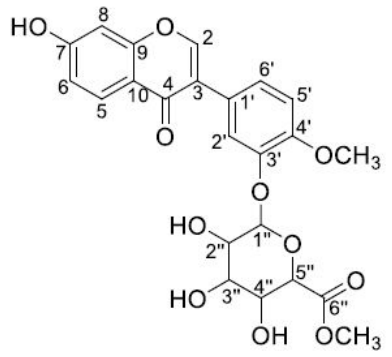

MI-3: Calycoisn-3'-O-glucuronide methyl ester

(c)

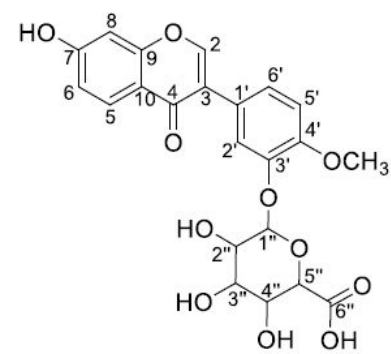

MI-2 (M32): Calycosin-3'-O -glucuronide

(b)

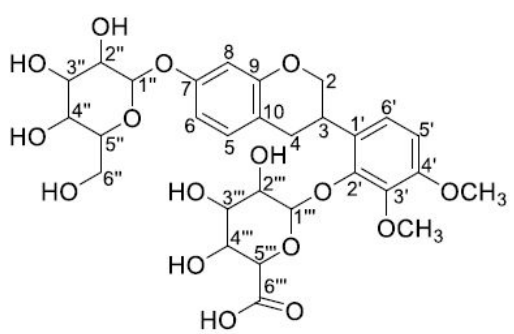

MI-4 (M106): Astraisoflavan-7-O-glucoside-2'-O-glucuronide

(d)

Figure 3. The structures of isolated metabolites from drug-containing urine. (a) MI-1 (M108); (b) MI-2 (M32); (c) MI-3; (d) MI-4 (M106).

MI-3 was obtained as a white powder and assigned a molecular formula of $\mathrm{C}_{23} \mathrm{H}_{22} \mathrm{O}_{11}$ based on its HR-ESI-MS mass spectrum, which showed a quasi-molecular ion peak [M - H] ${ }^{-}$at $m / z 473.1113$ (calcd. for $\mathrm{C}_{23} \mathrm{H}_{22} \mathrm{O}_{11}$ 473.1084). The main fragment ion was $m / z 283.0601\left[\mathrm{M}-\mathrm{C}_{7} \mathrm{H}_{10} \mathrm{O}_{6}-\mathrm{H}^{-}\right.$ in $\mathrm{MS}^{2}$ spectra, so it was predicted as a glucuronide methyl ester. ${ }^{1} \mathrm{H}-\mathrm{NMR}$ (DMSO-d $\left.6,400 \mathrm{MHz}\right)$ ppm: $8.31(1 \mathrm{H}, \mathrm{s}, \mathrm{H}-2), 7.97(1 \mathrm{H}, \mathrm{d}, J=8.7 \mathrm{~Hz}, \mathrm{H}-5), 6.94(1 \mathrm{H}, \mathrm{dd}, J=8.7 \mathrm{~Hz}, 2.2 \mathrm{~Hz}, \mathrm{H}-6), 6.87(1 \mathrm{H}$, $\mathrm{d}, J=2.2 \mathrm{~Hz}, \mathrm{H}-8), 7.29\left(1 \mathrm{H}, \mathrm{d}, J=2.0 \mathrm{~Hz}, \mathrm{H}-2^{\prime}\right), 7.05\left(1 \mathrm{H}, \mathrm{d}, J=8.5 \mathrm{~Hz}, \mathrm{H}-5^{\prime}\right), 7.23(1 \mathrm{H}, \mathrm{dd}, J=8.5 \mathrm{~Hz}$, 2.0Hz, H-6'), 3.79 (3H, s, C-4'-OCH $), 10.77(1 \mathrm{H}, \mathrm{s}, \mathrm{C}-7-\mathrm{OH}), 3.62\left(3 \mathrm{H}, \mathrm{s}, \mathrm{C}-6{ }^{\prime \prime}-\mathrm{OCH}_{3}\right) .{ }^{13} \mathrm{C}-\mathrm{NMR}$ (DMSO-d 6 , 100MHZ) ppm: 153.4 (C-2), 123.2 (C-3), 174.5 (C-4), 127.3 (C-5), 115.3 (C-6), 162.8 (C-7), 102.1 (C-8), 157.4 (C-9), 116.2 (C-10), 124.5 (C-1'), 116.6 (C-2'), 145.5 (C-3'), 149.0 (C-4'), 112.4 (C-5'), $123.0\left(\mathrm{C}-6^{\prime}\right), 55.8\left(\mathrm{C}-4^{\prime}-\mathrm{OCH}_{3}\right)$, were carbon signals of calycosin [31], $99.9\left(\mathrm{C}-1^{\prime \prime}\right), 73.0\left(\mathrm{C}-2^{\prime \prime}\right), 75.8$ $\left(\mathrm{C}-3^{\prime \prime}\right), 71.4\left(\mathrm{C}-4^{\prime \prime}\right), 75.2\left(\mathrm{C}-5^{\prime \prime}\right), 169.2$ (C-6") were carbon symbols of glucuronide, which were similar to those of calycosin-3'-O-glucuronide [31]. Compared with that, an additional methoxy carbon signal at $\delta 52.0$ was observed, and $\mathrm{H}$ signal of this methoxy at $\delta 3.62\left(3 \mathrm{H}, \mathrm{s}, \mathrm{C}-6^{\prime \prime}-\mathrm{OCH}_{3}\right)$ was in correlated with carbonyl carbon signal of glucuronide at $\delta 169.2$ (C-6") in HMBC spectra, indicating that the methoxy was linked to the carbonyl of glucuronide. According to all above analysis, MI-3 was identified as calycoisn-3'-O-glucuronide methyl ester (Figure 3c). It was a new compound. And its NMR spectroscopy was shown in Figure S28. But unfortunately, MI-3 could not be detected in the bio-samples using the HPLC-DAD-ESI-IT-TOF-MS ${ }^{n}$ technique. Therefore, MI-3 was maybe produced during the isolation process.

MI-4 (M106) was obtained as a faint yellow powder and assigned a molecular formula of $\mathrm{C}_{29} \mathrm{H}_{36} \mathrm{O}_{16}$ based on its HR-ESI-MS mass spectrum, which showed a quasi-molecular ion peak $[\mathrm{M}-\mathrm{H}]^{-}$at $m / z 639.1959$ (calcd. for $\mathrm{C}_{22} \mathrm{H}_{20} \mathrm{O}_{11}$ 639.1925). The main fragment ion was $m / z 463.1650$ $\left[\mathrm{M}-\mathrm{C}_{6} \mathrm{H}_{10} \mathrm{O}_{5}-\mathrm{H}\right]^{-}, 301.1066\left[\mathrm{M}-\mathrm{C}_{6} \mathrm{H}_{10} \mathrm{O}_{5}-\mathrm{C}_{6} \mathrm{H}_{8} \mathrm{O}_{8}-\mathrm{H}\right]^{-}$in $\mathrm{MS}^{2}$ spectra, so it was regarded as glucoside and glucuronide. ${ }^{1} \mathrm{H}-\mathrm{NMR}\left(\mathrm{DMSO}_{\mathrm{6}}, 400 \mathrm{MHz}\right) \mathrm{ppm}: 3.86(1 \mathrm{H}, \mathrm{t}, \mathrm{H}-2 \mathrm{a}), 4.23(1 \mathrm{H}, \mathrm{d}$, $J=8.0 \mathrm{~Hz}, \mathrm{H}-2 \mathrm{~b}), 3.59$ (1H, m, H-3), $2.74(1 \mathrm{H}, \mathrm{m}, \mathrm{H}-4 \mathrm{a}), 2.84(1 \mathrm{H}, \mathrm{m}, \mathrm{H}-4 \mathrm{~b}), 6.98(1 \mathrm{H}, \mathrm{d}, J=8.4 \mathrm{~Hz}$, H-5), $6.54(1 \mathrm{H}, \mathrm{dd}, J=8.4 \mathrm{~Hz}, 2.4 \mathrm{~Hz}, \mathrm{H}-6), 6.48(1 \mathrm{H}, \mathrm{d}, J=2.4 \mathrm{~Hz}, \mathrm{H}-8), 6.81\left(1 \mathrm{H}, \mathrm{d}, J=8.8 \mathrm{~Hz}, \mathrm{H}-5^{\prime}\right)$, 
$6.92\left(1 \mathrm{H}, \mathrm{d}, J=8.8 \mathrm{~Hz}, \mathrm{H}-6^{\prime}\right), 3.72\left(3 \mathrm{H}, \mathrm{s}, \mathrm{C}-3^{\prime}-\mathrm{OCH}_{3}\right), 3.77\left(3 \mathrm{H}, \mathrm{s}, \mathrm{C}-4^{\prime}-\mathrm{OCH}_{3}\right) .{ }^{13} \mathrm{C}-\mathrm{NMR}\left(\mathrm{DMSO}-\mathrm{d}_{6}\right.$, 100MHZ) ppm: 69.8 (C-2), 30.0 (C-3), 30.9 (C-4), 130.0 (C-5), 108.8 (C-6), 156.8 (C-7), 104.0 (C-8), 115.9 (C-9), 154.6 (C-10), 128.4 (C-1'), 147.2 (C-2'), 141.1 (C-3'), 152.1 (C-4'), 121.8 (C-5'), 103.2 (C-6'), $60.5\left(\mathrm{C}-3^{\prime}-\mathrm{OCH}_{3}\right), 55.8\left(\mathrm{C}-4^{\prime}-\mathrm{OCH}_{3}\right), 100.8\left(\mathrm{C}-1^{\prime \prime}\right), 73.7\left(\mathrm{C}-2^{\prime \prime}\right), 76.6\left(\mathrm{C}-3^{\prime \prime}\right), 69.5\left(\mathrm{C}-4^{\prime \prime}\right), 77.1\left(\mathrm{C}-5^{\prime \prime}\right)$, 60.8 (C-6") were carbon signal of astraisoflavan-7-O-glucoside [36]. And 100.9 (C-1"'), 73.3 (C-2"'), 75.8 (C-3"') 71.5 (C-4"'), $75.7\left(\mathrm{C}-5^{\prime \prime \prime}\right), 170.1$ (C-6"') were carbon signal of glucuronide. According to HMBC spectra, 84.90 , which was the terminal hydrogen signal of glucuronide, related to the $\delta 147.2$ (C-2'), which indicated that glucuronide was linked to C-2'. At the same time, the terminal hydrogen of glucoside $\delta 4.79$ was in correlated with glucoside carbon at $\delta 156.8$ (C-7), which indicated that glucoside was linked to C-7. Considering all of the above, MI-4 (M106) was determined as astraisoflavan-7-O-glucoside-2'-O-glucuronide (Figure 3d). It was a compound that isolated and identified by NMR for the first time. And its NMR spectroscopy was shown in Figure S29.

\subsection{ARTF-Related Pharmacological Effect of Compounds In Vivo}

The pharmacological literature of over 40 existence forms of ARTF which have specific or potential structure were retrieved from SciFinder and then analyzed. We found that 13 existence forms showed related pharmacological effect to ARTF, such as cardiovascular protective, neuroprotective, anti-inflammatory, and so on (Table S4). Six of them were original constituents, namely calycosin (F1), calycosin-7-O-glucoside (F4), ononin (F5), formononetin (F6), daidzein (F11), naringin (F18); seven of them were metabolites, namely daidzein-4'-O-sulfate (M107), daidzein-7-O-sulfate (M108), daidzein-7-O-glucuronide or daidzein-4'-O-glucuronide (M110), genistein (M118), genistein-7-O-sulfate and genistein-4'-O-sulfate (two of M119-M121), and equol-7-O-sulfate (M132 or M133). From Table S4, we could find that the metabolites in Table S4, especially phase II metabolites, could activate estrogen receptor (ER). ER activation is associated with cardiovascular protective [37] and anti-inflammatory [38] effects, which is the main pharmacological effect of ARTF. In addition, we predicted that sulfate of flavonoids might have an effect on ER by molecular docking technique (data not shown). Furthermore, phase II metabolites, especially sulfates, were the main existence forms of ARTF, so it could be speculated that some existence forms in vivo might be the material bases of the efficacies of ARTF, i.e., its effective forms.

\section{Materials and Methods}

\subsection{Chemicals and Materials}

ARTF (lot: 20170730) was obtained from Shanxi Baoji Herbest Biotech Co., Ltd. (Baoji, Shanxi, China) in August 2017 and its content was 62.7\%, and the content of six main flavonoids namely calycosin-7-O-glucoside, calycosin, ononin, formononetin, astrapterocarpan-3-O-glucoside, and asisoflavan-7-O-glucoside was $11.3 \%, 6.3 \%, 5.8 \%, 5.4 \%, 7.2 \%$, and $1.6 \%$, respectively, which was detected by HPLC-DAD-ELSD and calculated by using area normalization of ELSD (Evaporative Light Scattering Detector) chromatogram (Figure S30). The analysis of its constituents was conducted by HPLC-DAD-ESI-IT-TOF-MS ${ }^{\mathrm{n}}$, and 69 constituents had been identified (Table S5).

Calycosin (lot: MUST-16031110), calycosin-7-O-glucoside (lot: MUST-16031205) were brought from Chengdu Must Biotech Co., Ltd. (Chengdu, Sichuang, China). Formononetin (lot: LS60Q22) was supplied by Beijing J\&K Scientific Co., Ltd. (Beijing, China). Astrapterocarpan (lot: PRF7042221), astrapterocarpan-3-O-glucoside (lot: PRF7043003), astraisoflavan (lot: PRF7120221) were provided by Chengdu Biopurify Phytochemicals Ltd. (Chengdu, Sichuang, China). Astraisoflavan-7-O-glucoside (lot: 160217) was purchased from Chengdu Pufei De Biotech Co., Ltd. (Chengdu, Sichuang, China). Ononin was isolated in our laboratory [36]. The purities for all reference compounds were over $98 \%$.

Acetonitrile (HPLC grade, lot: 184866), formic acid (LC/MS grade, lot: 182088) were purchased from Thermo Fisher Scientific (Waltham, MA, USA). Methanol was supplied by Tianjing Damao Co., Ltd. (Tianjing, China). Sodium carboxymethyl cellulose (CMC-Na, Analytical grade) was purchased 
from Tianjing Guangfu Fine Chemical Research Institute (Tianjin, China). XAD-2 macroporous resins (lot: 94664), ODS (lot:9833), sephadex LH-20 were applied by Supelco (Bellefonte, PA, USA), YMC (Kyoto, Japan), and Amersham Biociences (Boston, MA, USA), respectively. Ultra-pure water was obtained by a Millipore Milli-Q Integral 3 Ultrapure water system (Billerica, MA, USA).

\subsection{Animals and ARTF Administration}

Fifty male Sprague-Dawley (SD) rats (250-300g) were obtained from the Experimental Animal Center of Peking University Health Science Center (Beijing, China) and 40 of them were kept in metabolic cages (Type: DXL-DL, Suzhou Fengshi Laboratory Animal Equipment Co. Ltd. (Suzhou, Jiangsu, China)) with two rats in each cage, and the other 10 rats were kept in normal cages, for free water and food twice a day. All the animals were maintained in an environmentally controlled breeding room for two days. Then, the next two days, urine and feces samples were collected twice a day and combined as blank samples. After that, the 40 rats in metabolic cages were orally administered with ARTF at $200 \mathrm{mg} / \mathrm{kg}$ (the suspension of ARTF at $25 \mathrm{mg} / \mathrm{kg}$ was prepared with $0.5 \%$ CMC-Na in an ultrasonic bath), twice a day (8:00 am and 8:00 pm) for 31 days, and the 10 rats in normal cages weren't administered anything except for water and food. The animal experiments were approved by the Biomedical Ethical Committee of Peking University (approval No. LA2016205).

\subsection{Bio-Samples Collection and Pre-Treatment}

\subsubsection{Urine Collection and Pre-Treatment}

Urine samples were collected twice a day (8:00 am and 8:00 pm) after administration of ARTF from metabolic cages and merged together, then filtered to remove impurities such as hair and dried in vacuum at $50{ }^{\circ} \mathrm{C}$ using a Heidolph Laborota 4001 rotatory evaporator (Heidolph Instruments $\mathrm{GmhH}$ \& Co., Schwabach, Germany). After that, at a ratio of $1.0 \mathrm{~g}$, dried samples were reconstituted in $10 \mathrm{~mL}$ methanol followed by 30 min ultrasonic extraction and filtered, then the filtrate was dried in vacuum (ca $25 \mathrm{~g} /$ day obtained) and $1.0 \mathrm{~g}$ sample was added $1 \mathrm{~mL}$ methanol to resuspended and stored at $-20^{\circ} \mathrm{C}$. After 31 days, all the processed urine samples were mixed together as one sample and dried in vacuum. g). $1.0 \mathrm{~g}$ of the urine extract was taken out and redissolved in $5 \mathrm{~mL}$ methanol, then centrifuged at 15,000 rpm for $15 \mathrm{~min}$ and the supernatant was filtered through $0.45 \mu \mathrm{m}$ nylon filter (Tianjin jinteng Experiment Co. Ltd., Tianjin, China). Finally, the filtrate was transferred into sample injection vial waiting for LC/MS analysis.

\subsubsection{Feces Collection and Pre-Treatment}

Feces samples were collected twice a day (8:00 am and 8:00 pm) from metabolic cages. Feces samples of each day were dried in $50^{\circ} \mathrm{C}$ for $48 \mathrm{~h}$ and were crushed into powder. A pulverized sample of $1.0 \mathrm{~g}$ was extracted with $5 \mathrm{~mL}$ methanol for $30 \mathrm{~min}$ in an ultrasonic bath three times. Afterward, the filtrates were combined and concentrated to dryness, then redissolved in 10 folds methanol to store at $-20^{\circ} \mathrm{C}$. All feces samples were combined after processing, and concentrated to dryness as ARTF-containing feces extract; $0.3 \mathrm{~g}$ was taken out to resuspended in $3 \mathrm{~mL}$ methanol and centrifuged at 15,000 rpm for $15 \mathrm{~min}$, and filtered through $0.45 \mu \mathrm{m}$ nylon filter before LC/MS analysis.

\subsubsection{Plasma Collection and Pre-Treatment}

At 32nd day, blood samples from rats in metabolic cages were collected by heart puncture technique under anesthesia at $10 \mathrm{~min}, 30 \mathrm{~min}, 1 \mathrm{~h}, 2 \mathrm{~h}$, and $4 \mathrm{~h}$ (eight rats were sacrificed at each time point), and centrifuged at $5000 \mathrm{rpm}$ for $15 \mathrm{~min}$ to obtain ARTF-containing plasma. Blank plasma was collected and processed in the same way from the rats in normal cages. All ARTF-containing and blank plasma were merged respectively, and 10 folds volume of methanol was added, and ultrasonically vibrated for $30 \mathrm{~min}$ to precipitate protein. Then, the mixture was centrifuged at $5000 \mathrm{rpm}$ for $15 \mathrm{~min}$, and the supernatant was condensed and dissolved in methanol, and the volume of methanol was $1 \%$ 
of the initial volume of plasma, and then centrifuged at 15,000 rpm for $15 \mathrm{~min}$, finally filtered through $0.45 \mu \mathrm{m}$ nylon filter before LC/MS analysis.

\subsubsection{Organs Collection and Pre-Treatment}

After blood collection, the heart, liver, spleen, lung, kidney, stomach, small intestine, colon, thymus, and brain were quickly removed and flushed clearly with stroke-physiological saline solution until there was no obvious blood or content in the surface or cavity. All the organs were stored at $-80^{\circ} \mathrm{C}$. All the organs were shredded and suspended in deionized water at a ratio of $1.0 \mathrm{~g}$ to $4 \mathrm{~mL}$, then homogenized by ultrasound homogenizer (Ultra-Turrax T8, Ika-werke Gmbh \& Co. KG, Staufen, Germany). After that, $8 \mathrm{~mL}$ homogenates were extracted with 10 -folds volume methanol in an ultrasonic bath for $30 \mathrm{~min}$. The mixture was centrifuged at $5000 \mathrm{rpm}$ for $15 \mathrm{~min}$, and the supernatant was separated, dried, and resuspended in $2 \mathrm{~mL}$ methanol and filtered through $0.45 \mu \mathrm{m}$ nylon filter before LC/MS analysis.

\subsection{Isolation and Identification of Metabolites from ARTF-Containing Urine}

Isolation procedure: ARTF-containing urine extract obtained in Section 3.3 (ca. 750 g), was dissolved in $1.5 \mathrm{~L}$ deionized water, filtered, and then subjected to XAD-2 macroporous resins column chromatography. Water, $20 \%$ methanol-water, $60 \%$ methanol-water, and $100 \%$ methanol were used to elute the column and get Fraction 1 to Fraction 4, respectively. The four metabolites, MI-1 (M108) (2.67 mg), MI-2 (M32) (818.30 mg), MI-3 (16.66 mg), MI-4 (M106) (34.62 mg), were isolated and purified from Fraction-3 by ODS column chromatography, Sephadex LH-20 column chromatography, and a Shimadzu preparative HPLC system sequentially, and their purity was above $90 \%$ determined by an Agilent 1200 HPLC.

Structure identification using NMR: MI-1 and the other three metabolites were dissolved in $0.15 \mathrm{~mL}$ and $0.5 \mathrm{~mL}$ DMSO-d6, respectively. Their ${ }^{1} \mathrm{H},{ }^{13} \mathrm{C}$, heteronuclear singular quantum correlation (HSQC), heteronuclear multiple bond correlation (HMBC). NMR spectra were recorded on a Bruker DRX-400 NMR spectrometer (Bruker, Rheinstetten, Germany), using tetramethylsilane (TMS) as internal standard. All chemical shifts were reported in parts per million (ppm, $\delta$ ), and coupling constants $(J)$ in Hertz. UV spectra $(200-400 \mathrm{~nm})$ and HRMS data were recorded on the LC-MS-IT-TOF instrument with a PDA detector.

\subsection{Instruments and Conditions}

HPLC-DAD-ESI-IT-TOF-MS ${ }^{n}$ analyses were performed on a Shimadzu HPLC instrument (two LC-20AD pumps, an SIL-20AC autosampler, a CTO-20A column oven, an SPD-M20A PDA detector, a CBM-20A system controller) coupled with an IT-TOF mass spectrometer (Shimadzu, Kyoto, Japan) through an ESI interface. All data were processed by Shimadzu software, specifically, LCMS solution Version 3.60, Formula Predictor Version 1.2. and Accurate Mass Calculator. The chromatography separations were performed on an Industries Epic $\mathrm{C}_{18}$ column $(250 \mathrm{~mm} \times 4.6 \mathrm{~mm}, 5 \mu \mathrm{m})(\mathrm{New}$ Brunswick, NJ, USA) protected with an Agilent ZORBAX SB C 18 column $(12.5 \mathrm{~mm} \times 4.6 \mathrm{~mm}, 5 \mu \mathrm{m})$ (Santa Clara, CA, USA). The mobile phase consisted of water-formic acid (100:0.1, $v / v)(A)$ and acetonitrile (B) at a flow rate of 10,000 $\mathrm{mL} / \mathrm{min}$. A gradient elution program was adopted, specifically as $5 \% \mathrm{~B}$ at $0-10 \mathrm{~min}$, $5-16 \%$ B at $10-12 \mathrm{~min}, 16-20 \%$ B at $12-25 \mathrm{~min}, 20-22 \%$ B at $25-45 \mathrm{~min}, 22-35 \%$ B at $45-60 \mathrm{~min}$, $35-60 \% \mathrm{~B}$ at $60-85 \mathrm{~min}, 60-100 \%$ at $85-90 \mathrm{~min}$. At the end of each run, $100 \% \mathrm{~B}$ was used to flush the column for $20 \mathrm{~min}$. For mass detection, the mass spectrometer was programmed to carry out a full scan over $\mathrm{m} / \mathrm{z}$ 100-1500 (MS ${ }^{1}$ ) with $30 \mathrm{~ms}$ accumulation time and $\mathrm{m} / \mathrm{z}$ 50-1500 (MS ${ }^{2}$ and $\mathrm{MS}^{3}$ ) with $20 \mathrm{~ms}$ accumulation in both positive ion (PI) and negative ion (NI) detection mode; the flow rate was $0.2000 \mathrm{~mL} / \mathrm{min}$; the heat block and curved desolvation line temperature was $200^{\circ} \mathrm{C}$; the nebulizing nitrogen gas flow was $1.5 \mathrm{~L} / \mathrm{min}$; the interface voltage was $(+), 4.5 \mathrm{kV} ;(-), 3.5 \mathrm{kV}$; the detector voltage was $1.7 \mathrm{kV}$; the relative collision-induced dissociation energy was $50 \%$. 


\section{Conclusions}

In summary, 170 kinds of compound (23 original constituents and 147 metabolites) were identified after administration of ARTF to rats, which included three newly detected original constituents and 89 new metabolites of ARTF, and 12 were regarded as new compounds (they are all metabolites) by retrieving information from the Sci inder database. Nineteen original constituents and 65 metabolites were detected and characterized in 10 organs. Four metabolites, including a new compound (calycoisn-3'-O-glucuronide methyl ester) and a first-isolated compound (astraisoflavan-7-O-glucoside-2'-O-glucuronide), along with two known compounds (daidzein-7-O-sulfate and calycosin-3'-O-glucuronide) were isolated from ARTF-containing urine and identified by NMR. Although the bioactivity studies of phase II metabolites were little, 13 compounds (six original constituents, one phase I metabolite, six phase II metabolites) in vivo were reported to possess similar pharmacological effects with ARTF, which indicated that they were effective forms of ARTF, and phase II metabolites might contribute to the efficacies of ARTF in vivo.

In the future, firstly, more kinds of phase I and phase II metabolites of ARTF should be obtained by synthesis or biotransformation. Then, the bioactivities of these metabolites should be determined to clarify the effective forms of ARTF. After that, the action mechanism of the effective forms can be studied. Finally, a new strategy to evaluate and control the quality of AR can be established.

Supplementary Materials: The following are available online. Detailed information on the determination of the contents of ARTF and its major constituents and the isolation procedure of compounds from urine, with Tables S1-S5 and Figures S1-S35 are available. References [39-69] are cited in the Supplementary Materials.

Author Contributions: Conceptualization, S.-Q.C. and F.X.; methodology, F.X., H.-F.L.; validation, S.-Q.C., F.X.; formal analysis, L.-J.L.; investigation, L.-J.L., H.-Y.W., Y.-F.Z.; writing-original draft preparation, L.-J.L; writing-review and editing, L.-J.L., H.-F.L., F.X., G.-X.L., M.-Y.S., X.W., S.-Q.C.; supervision, S-Q.C., F.X.; project administration, S-Q.C., F.X., L.-J.L.; funding acquisition, S.-Q.C., F.X. All authors have read and agreed to the published version of the manuscript.

Funding: This research was funded by the National Natural Science Foundation of China (NO. 81673595) and the National Science and Technology Major Project for Significant New Drugs Development (2019ZX09201004).

Conflicts of Interest: The authors declare no conflict of interest.

\section{References}

1. Jing, L.; Zhao, Z.Z.; Chen, H.B. Review of Astragali Radix. Chin. Herb. Med. 2011, 3, 90-105.

2. Wang, D.Q.; Zhuang, Y.; Tian, Y.P.; Thomas, G.N.; Ying, M.Z.; Tomlinson, B. Study of the effects of total flavonoids of Astragalus on atherosclerosis formation and potential mechanisms. Oxid. Med. Cell Longev. 2012, 2012, 1-10. [CrossRef] [PubMed]

3. Wang, D.Q.; Ding, B.G.; Tomas, G.N.; Tian, Y.P.; Wang, C.B.; Tomlinson, B. Effects of total flavonoids of astragalus on early atheroselerosis in rabbits with diet-induced hyPereholesterolemia. Chin. Pharmacol. Bull. 2003, 19, 637-639.

4. Liu, Q.; Zhang, L.; Shan, Q.; Ding, Y.; Zhang, Z.; Zhu, M.; Mao, Y. Total flavonoids from Astragalus alleviate endothelial dysfunction by activating the Akt/eNOS pathway. J. Int. Med. Res. 2018, 46, 2096-2103. [CrossRef]

5. Li, Q. Effects of Total Flavonoids of Astragalus on Inflammatory Response in Brain Tissue of Natural Aging Rats and Its Related Mechanism. J. Beihua Univ. (Nat. Sci.) 2018, 19, 745-749.

6. Guo, Z.; Xu, H.Y.; Xu, L.; Wang, S.S.; Zhang, X.M. In Vivo and in Vitro Immunomodulatory and Anti-Inflammatory Effects of Total Flavonoids of Astragalus. Afr. J. Tradit. Complement. Altern. Med. 2016, 13, 60-73. [CrossRef]

7. Li, J.; Xu, L.; Sang, R.; Yu, Y.; Ge, B.; Zhang, X. Immunomodulatory and anti-inflammatory effects of total flavonoids of Astragalus by regulating NF- $\mathrm{B}$ and MAPK signalling pathways in RAW 264.7 macrophages. Pharmazie 2018, 73, 589-593.

8. Wang, D.Q.; Ding, B.G.; Ma, Y.Q.; Zhao, H.L.; Thomas, G.N.; Tomlinson, B.; Tian, Y.P.; Wang, C.B.; Critchley, J.A. Studies on Protective Effect of Total Flavonoids of Astragalus on Liver Damage Paracetamol-Induced by. Chin. J. Chin. Mater. Med. 2001, 26, 483-486. 
9. Shen, W.M.; Wang, C.B.; Wang, D.Q.; Tian, Y.P.; Yan, G.T.; Hao, X.H. The protective effects of TFA on reperfusion induced hepatic injury in hemorrhagic shock. Chin. Pharmacol. Bull. 1997, 13, 532-534.

10. Lee, E.J.; Yean, M.H.; Jung, H.S.; Kim, J.S.; Kang, S.S. Phytochemical Studies on Astragalus Root (2); Flavonoids and a Lignan. Nat. Prod. Sci. 2008, 14, 131-137.

11. Yang, L.P.; Shen, J.G.; Xu, W.C.; Li, J.; Jiang, J.Q. Secondary metabolites of the genus Astragalus: Structure and biological-activity update. Chem. Biodivers. 2013, 10, 1004-1054. [CrossRef] [PubMed]

12. Zhang, L.J.; Liu, H.K.; Hsiao, P.C.; Kuo, L.M.Y.; Lee, I.J.; Wu, T.S.; Chiou, W.F.; Kuo, Y.H. New Isoflavonoid Glycosides and Related Constituents from Astragali Radix (Astragalus membranaceus) and Their Inhibitory Activity on Nitric Oxide Production. J. Agr. Food Chem. 2011, 59, 1131-1137. [CrossRef] [PubMed]

13. Yan, Z.; Lin, G.; Ye, Y.; Wang, Y.; Yan, R. A generic multiple reaction monitoring based approach for plant flavonoids profiling using a triple quadrupole linear ion trap mass spectrometry. J. Am. Soc. Mass. Spectr. 2014, 25, 955-965. [CrossRef]

14. Zhang, Y.Z.; Xu, F.; Yang, D.H.; Shang, M.Y.; Yang, D.H.; Wang, X.; Cai, S.Q. Profiling and identification of the metabolites of calycosin in rat hepatic $9000 \times \mathrm{g}$ supernatant incubation system and the metabolites of calycosin-7-O- $\beta$-D-glucoside in rat urine by HPLC-DAD-ESI-IT-TOF-MSn technique. J. Pharm. Biomed. Anal. 2012, 70, 425-439. [CrossRef] [PubMed]

15. Zhang, Y.Z. Metabolic research of 8 isoflavone in Astragalus. Ph.D. Thesis, Beijing University, Beijing, China, 2011.

16. Zhang, Y.Z.; Xu, F.; Dong, J.; Liang, J.; Hashi, Y.; Liu, G.X.; Li, Y.L.; Shang, M.Y.; Wang, X.; Cai, S.Q. Profiling the metabolites of astrapterocarpan in rat hepatic $9000 \mathrm{~g}$ supernatant. Chin. J. Nat. Med. 2019, 17, 842-857. [CrossRef]

17. Hu, G.; Siu, S.O.; Li, S.; Chu, I.K.; Kwan, Y.W.; Chan, S.W.; Leung, G.P.H.; Yan, R.; Lee, S.M.Y. Metabolism of calycosin, an isoflavone from Astragali Radix, in zebrafish larvae. Xenobiotica 2012, 42, 294-303. [CrossRef]

18. Zhang, W.; Jiang, S.; Qian, D.W.; Shang, E.X.; Duan, J.A. Analysis of interaction property of calycosin-7-O- $\beta$-D-glucoside with human gut microbiota. J. Chromatogr. B Anal. Technol. Biomed. Life Sci. 2014, 963, 16-23. [CrossRef]

19. Davies, H.L.; Hill, J.L. The Effect of Diet on the Metabolism in Sheep of the Tritiated Isoflavones Formononetin and Biochanin-A. Aust. J. Agr. Res. 1989, 40, 157-163. [CrossRef]

20. Tolleson, W.H.; Doerge, D.R.; Churchwell, M.I.; Marques, M.M.; Roberts, D.W. Metabolism of biochanin A and formononetin by human liver microsomes in vitro. J. Agr. Food Chem. 2002, 50, 4783-4790. [CrossRef]

21. Zhang, W.; Jiang, S.; Qian, D.W.; Shang, E.X.; Guan, H.L.; Ren, H.; Zhu, Z.H.; Duan, J.A. The interaction between ononin and human intestinal bacteria. Acta Pharm. Sinica 2014, 49, 1162-1168.

22. Li, H.F. Studies on the Metabolism of Astragali Radix In Vivo. Ph.D. Thesis, Shenyang Pharmaceutical University, Shenyang, China, 2017.

23. Sun, J. Progress and Application of Astragalus and its classic prescription. Chin. Hosp. Pharm. J. 2019, 39, 1311-1314.

24. Yang, P. Study on the In Vivo Metabolism of the Rhizomes of Smilax Glabra and Screening of Its Bioactive Components In Vivo. Ph.D. Thesis, Beijing University, Beijing, China, 2016.

25. Liang, J.; Xu, F.; Zhang, Y.Z.; Zang, X.Y.; Wang, D.; Shang, M.Y.; Wang, X.; Chui, D.H.; Cai, S.Q. The profiling and identification of the metabolites of (+)-catechin and study on their distribution in rats by HPLC-DAD-ESI-IT-TOF-MS(n) technique. Biomed. Chromatogr. 2014, 28, 401-411. [CrossRef] [PubMed]

26. Liang, J.; Xu, F.; Zhang, Y.Z.; Huang, S.; Zang, X.Y.; Zhao, X.; Zhang, L.; Shang, M.Y.; Yang, D.H.; Wang, X.; et al. The profiling and identification of the absorbed constituents and metabolites of Paeoniae Radix Rubra decoction in rat plasma and urine by the HPLC-DAD-ESI-IT-TOF-MS(n) technique: A novel strategy for the systematic screening and identification of absorbed constituents and metabolites from traditional Chinese medicines. J. Pharm. Biomed. Anal. 2013, 83, 108-121. [PubMed]

27. Gao, X.; Mu, J.; Li, Q.; Guan, S.; Liu, R.; Du, Y.; Zhang, H.; Bi, K. Comprehensive Identification of Guan-Xin-Shu-Tong Capsule via a Mass Defect and Fragment Filtering Approach by High Resolution Mass Spectrometry: In Vitro and In Vivo Study. Molecules 2017, 22, 1007. [CrossRef]

28. Zhao, X.; Yang, D.H.; Xu, F.; Huang, S.; Zhang, L.; Liu, G.X.; Cai, S.Q. The in vivo absorbed constituents and metabolites of Danshen decoction in rats identified by HPLC with electrospray ionization tandem ion trap and time-of-flight mass spectrometry. Biomed. Chromatogr. 2015, 29, 285-304. [CrossRef] 
29. Li, W.L.; Sun, X.M.; Song, H.; Ding, J.X.; Bai, J.; Chen, Q. HPLC/Q-TOF-MS-Based Identification of Absorbed Constituents and Their Metabolites in Rat Serum and Urine after Oral Administration of Cistanche deserticola Extract. J. Food Sci. 2015, 80, H2079-H2087. [CrossRef]

30. Hamed, A.I.; Ben Said, R.; Kontek, B.; Al-Ayed, A.S.; Kowalczyk, M.; Moldoch, J.; Stochmal, A.; Olas, B. LC-ESI-MS/MS profile of phenolic and glucosinolate compounds in samh flour (Mesembryanthemum forsskalei Hochst. ex Boiss) and the inhibition of oxidative stress by these compounds in human plasma. Food Res. Int. 2016, 85, 282-290. [CrossRef]

31. Ren, X.L. Study on the Effective Substances of Buyang Huanwu Decoction-Study on the Chemical Constituents of Buyang Huanwu Decoction in Pigs Urine. Master's Thesis, Beijing University, Beijing, China, 2013.

32. Fang, G.; Zhang, P.; Ye, X.L. Elecron spray ion trap mass spectrometry of isoflavones and isoflavone aglycones of Semen Sojae Praeparatum. Acad. J. Second Mil. Med. Univ. 2013, 34, 1108-1115. [CrossRef]

33. Antignac, J.P.; Cariou, R.; Le Bizec, B.; Andre, F. New data regarding phytoestrogens content in bovine milk. Food Chem. 2004, 87, 275-281. [CrossRef]

34. Murthy, M.S.R.; Rao, E.V.; Ward, R.S. Carbon-13 nuclear magnetic resonance spectra of isoflavones. Magn. Reson. Chem. 1986, 24, 225-230. [CrossRef]

35. Nakano, H.; Ogura, K.; Takahashi, E.; Harada, T.; Nishiyama, T.; Muro, K.; Hiratsuka, A.; Kadota, S.; Watabe, T. Regioselective monosulfation and disulfation of the phytoestrogens daidzein and genistein by human liver sulfotransferases. Drug Metab. Pharmacol. 2004, 19, 216-226. [CrossRef] [PubMed]

36. Zhang, Y.Z.; Xu, F.; Liang, J.; Tang, J.S.; Shang, M.Y.; Wang, X.; Cai, S.Q. Isoflavonoids from roots of Astragalus membranaceus var. mongholicus. Chin. J. Chin. Mater. Med. 2012, 37, 3243-3248.

37. Knowlton, A.A.; Lee, A.R. Estrogen and the cardiovascular system. Pharmacol. Ther. 2012, 135, 54-70. [CrossRef]

38. Straub, R.H. The complex role of estrogens in inflammation. Endocr. Rev. 2007, 28, 521-574. [CrossRef] [PubMed]

39. Bai, F.; Makino, T.; Kono, K.; Nagatsu, A.; Ono, T.; Mizukami, H. Calycosin and formononetin from astragalus root enhance dimethylarginine dimethylaminohydrolase 2 and nitric oxide synthase expressions in Madin Darby Canine Kidney II cells. J. Nat. Med. 2013, 67, 782-789. [CrossRef]

40. Yu, D.H.; Bao, Y.M.; An, L.J.; Yang, M. Protection of PC12 Cells against Superoxide-induced Damage by Isoflavonoids from Astragalus mongholicus. Biomed. Environ. Sci. 2009, 22, 50-54. [CrossRef]

41. Ma, R.; Yuan, F.; Wang, S.; Liu, Y.; Fan, T.; Wang, F. Calycosin alleviates cerulein-induced acute pancreatitis by inhibiting the inflammatory response and oxidative stress via the p38 MAPK and NF-kappaB signal pathways in mice. Biomed. Pharmacother. 2018, 105, 599-605. [CrossRef]

42. Tian, J.; Wang, Y.; Zhang, X.; Ren, Q.Y.; Li, R.; Huang, Y.; Lu, H.L.; Chen, J. Calycosin inhibits the in vitro and in vivo growth of breast cancer cells through WDR7-7-GPR30 Signaling. J. Exp. Clin. Canc. Res. 2017, 36, 153-166. [CrossRef]

43. Duan, X.; Meng, Q.; Wang, C.; Liu, Z.; Sun, H.; Huo, X.; Sun, P.; Ma, X.; Peng, J.; Liu, K. Effects of calycosin against high-fat diet-induced nonalcoholic fatty liver disease in mice. J. Gastroenterol Hepatol 2018, 33, 533-542. [CrossRef]

44. Ren, M.; Wang, X.; Du, G.; Tian, J.; Liu, Y. Calycosin-7-O- $\beta$-D-glucoside attenuates ischemia-reperfusion injury in vivo via activation of the PI3K/Akt pathway. Mol. Med. Rep. 2015, 13, 633-640. [CrossRef]

45. Fu, S.P.; Gu, Y.; Jiang, J.Q.; Chen, X.; Xu, M.J.; Chen, X.M.; Shen, J.G. Calycosin-7-O-beta-D-glucoside regulates nitric oxide/caveolin-1/matrix metalloproteinases pathway and protects blood-brain barrier integrity in experimental cerebral ischemia-reperfusion injury. J. Ethnopharmacol. 2014, 155, 692-701. [CrossRef] [PubMed]

46. Choi, S.I.; Heo, T.R.; Min, B.H.; Cui, J.H.; Choi, B.H.; Park, S.R. Alleviation of osteoarthritis by calycosin-7-O- $\beta$-D-glucopyranoside (CG) isolated from Astragali radix (AR) in rabbit osteoarthritis (OA) model. Osteoarthritis Cartilage 2007, 15, 1086-1092. [CrossRef] [PubMed]

47. Zhang, D.M. Effect of calycosin-7-O-beta-D-glucoside on cell apoptosis in cervical cancer HeLa cell and expression of Bcl-2/Bax. Chin. Tradit. Herbal Drugs 2015, 46, 1498-1502. 
48. Zhu, J.T.T.; Choi, R.C.Y.; Chu, G.K.Y.; Cheung, A.W.H.; Gao, Q.T.; Li, J.; Jiang, Z.Y.; Dong, T.T.X.; Tsim, K.W.K. Flavonoids possess neuroprotective effects on cultured pheochromocytoma PC12 cells: A comparison of different flavonoids in activating estrogenic effect and in preventing $\beta$-amyloid-induced cell death. J. Agr Food Chem. 2007, 55, 2438-2445. [CrossRef]

49. Dong, L.; Yin, L.; Zhang, Y.; Fu, X.; Lu, J. Anti-inflammatory effects of ononin on lipopolysaccharide-stimulated RAW 264.7 cells. Mol. Immunol. 2017, 83, 46-51. [CrossRef]

50. Jun, M.; Hong, J.; Jeong, W.S.; Ho, C.T. Suppression of arachidonic acid metabolism and nitric oxide formation by kudzu isoflavones in murine macrophages. Mol. Nutr. Food Res. 2005, 49, 1154-1159. [CrossRef]

51. Jin, Y.M.; Xu, T.M.; Zhao, Y.H.; Wang, Y.C.; Cui, M.H. In vitro and in vivo anti-cancer activity of formononetin on human cervical cancer cell line HeLa. Tumour Biol. 2014, 35, 2279-2284. [CrossRef]

52. Yang, S.; Wei, L.; Xia, R.; Liu, L.; Chen, Y.; Zhang, W.; Li, Q.; Feng, K.; Yu, M.; Zhang, W.; et al. Formononetin ameliorates cholestasis by regulating hepatic SIRT1 and PPARalpha. Biochem. Biophys. Res. Commun. 2019, 512, 770-778. [CrossRef]

53. Applova, L.; Karlickova, J.; Riha, M.; Filipsky, T.; Macakova, K.; Spilkova, J.; Mladenka, P. The isoflavonoid tectorigenin has better antiplatelet potential than acetylsalicylic acid. Phytomedicine 2017, 35, 11-17. [CrossRef]

54. Valeri, A.; Fiorenzani, P.; Rossi, R.; Aloisi, A.M.; Valoti, M.; Pessina, F. The soy phytoestrogens genistein and daidzein as neuroprotective agents against anoxia-glucopenia and reperfusion damage in rat urinary bladder. Pharmacol Res. 2012, 66, 309-316. [CrossRef]

55. He, Y.; Wu, X.; Cao, Y.; Hou, Y.; Chen, H.; Wu, L.; Lu, L.; Zhu, W.; Gu, Y. Daidzein exerts anti-tumor activity against bladder cancer cells via inhibition of FGFR3 pathway. Neoplasma 2016, 63, 523-531. [CrossRef] [PubMed]

56. Wong, M.C.; Portmann, B.; Sherwood, R.; Niemela, O.; Koivisto, H.; Parkkila, S.; Trick, K.; L'Abbe, M.R.; Wilson, J.; Dash, P.R.; et al. The cytoprotective effect of alpha-tocopherol and daidzein against d-galactosamine-induced oxidative damage in the rat liver. Metabolism 2007, 56, 865-875. [CrossRef] [PubMed]

57. Rajadurai, M.; Prince, P.S. Preventive effect of naringin on isoproterenol-induced cardiotoxicity in Wistar rats: An in vivo and in vitro study. Toxicology 2007, 232, 216-225. [CrossRef] [PubMed]

58. Golechha, M.; Chaudhry, U.; Bhatia, J.; Saluja, D.; Arya, D.S. Naringin protects against kainic acid-induced status epilepticus in rats: Evidence for an antioxidant, anti-inflammatory and neuroprotective intervention. Biol. Pharm. Bull. 2011, 34, 360-365. [CrossRef] [PubMed]

59. Li, J.; Dong, Y.; Hao, G.; Wang, B.; Wang, J.; Liang, Y.; Liu, Y.; Zhen, E.; Feng, D.; Liang, G. Naringin suppresses the development of glioblastoma by inhibiting FAK activity. J. Drug Target. 2017, 25, 41-48. [CrossRef] [PubMed]

60. Zhou, C.; Lai, Y.; Huang, P.; Xie, L.; Lin, H.; Zhou, Z.; Mo, C.; Deng, G.; Yan, W.; Gao, Z.; et al. Naringin attenuates alcoholic liver injury by reducing lipid accumulation and oxidative stress. Life Sci. 2019, 216, 305-312. [CrossRef]

61. Guo, D.; Wang, J.; Wang, X.; Luo, H.; Zhang, H.; Cao, D.; Chen, L.; Huang, N. Double directional adjusting estrogenic effect of naringin from Rhizoma drynariae (Gusuibu). J. Ethnopharmacol. 2011, 138, 451-457. [CrossRef]

62. Pugazhendhi, D.; Watson, K.A.; Mills, S.; Botting, N.; Pope, G.S.; Darbre, P.D. Effect of sulphation on the oestrogen agonist activity of the phytoestrogens genistein and daidzein in MCF-7 human breast cancer cells. J. Endocrinol. 2008, 197, 503-515. [CrossRef]

63. Kgomotso, T.; Chiu, F.; Ng, K. Genistein- and daidzein 7-O-beta-D-glucuronic acid retain the ability to inhibit copper-mediated lipid oxidation of low density lipoprotein. Mol. Nutr. Food Res. 2008, 52, 1457-1466. [CrossRef]

64. Morito, K.; Aomori, T.; Hirose, T.; Kinjo, J.; Hasegawa, J.; Ogawa, S.; Inoue, S.; Muramatsu, M.; Masamune, Y. Interaction of phytoestrogens with estrogen receptors $\alpha$ and $\beta$ (II). Biol. Pharm. Bull. 2002, 25, 48-52. [CrossRef]

65. Farina, H.G.; Pomies, M.; Alonso, D.F.; Gomez, D.E. Antitumor and antiangiogenic activity of soy isoflavone genistein in mouse models of melanoma and breast cancer. Oncol. Rep. 2006, 16, 885-891. [CrossRef] [PubMed] 
66. Huang, Q.; Huang, R.; Zhang, S.; Lin, J.; Wei, L.; He, M.; Zhuo, L.; Lin, X. Protective effect of genistein isolated from Hydrocotyle sibthorpioides on hepatic injury and fibrosis induced by chronic alcohol in rats. Toxicol. Lett. 2013, 217, 102-110. [CrossRef] [PubMed]

67. Schrader, C.; Ernst, I.M.; Sinnecker, H.; Soukup, S.T.; Kulling, S.E.; Rimbach, G. Genistein as a potential inducer of the anti-atherogenic enzyme paraoxonase-1: Studies in cultured hepatocytes in vitro and in rat liver in vivo. J. Cell Mol. Med. 2012, 16, 2331-2341. [CrossRef] [PubMed]

68. Wan, Y.F.; XU, Y.J.; YUAN, B.; WANG, L.L.; Lin, L.Q.; Xu, H.Y. Synthesis of genistein sulfates and effects of genistein and its sulfates on proliferation of breast cancer cells. Shizhen Guoyi Guoyao 2011, 22, 284-286.

69. Rimbach, G.; Weinberg, P.D.; de Pascual-Teresa, S.; Alonso, M.G.; Ewins, B.A.; Turner, R.; Minihane, A.M.; Botting, N.; Fairley, B.; Matsugo, S.; et al. Sulfation of genistein alters its antioxidant properties and its effect on platelet aggregation and monocyte and endothelial function. Biochim. Biophys. Acta. 2004, 1670, 229-237. [CrossRef] [PubMed]

Sample Availability: Samples of the compounds are not available from the authors.

Publisher's Note: MDPI stays neutral with regard to jurisdictional claims in published maps and institutional affiliations.

(C) 2020 by the authors. Licensee MDPI, Basel, Switzerland. This article is an open access article distributed under the terms and conditions of the Creative Commons Attribution (CC BY) license (http://creativecommons.org/licenses/by/4.0/). 